
\title{
BResarch Soute \\ The Synchronization and Synchronization Transition of Coupled Fractional-order Neuronal Networks
}

\section{Xin Yang ( $\nabla$ y_angx_in@163.com )}

Air Force Engineering University

GuangJun Zhang

Air Force Engineering University https://orcid.org/0000-0001-8600-1845

XueRen Li

Air Force Engineering University

Dong Wang

Air Force Engineering University

\section{Research Article}

Keywords: Fractional-order , coupled neuronal network , electromagnetic radiation , synchronization transition

Posted Date: May 4th, 2021

DOI: https://doi.org/10.21203/rs.3.rs-458795/v1

License: (1) (i) This work is licensed under a Creative Commons Attribution 4.0 International License. Read Full License 


\title{
The synchronization and synchronization transition of coupled fractional-order neuronal networks
}

\author{
Xin Yang · GuangJun Zhang · XueRen Li · Dong Wang
}

Received: date / Accepted: date

\begin{abstract}
Different from the previous researches on the synchronization and synchronization transition of neuronal networks constructed by integer-order neuronal models, the synchronization and synchronization transition of fractional-order neuronal network are investigated in this paper. The fractional-order ring neuronal network constructed by fractional-order HindmarshRose (HR) neuronal models without electromagnetic radiation are proposed, and it's synchronization behaviors are investigated numerically. The synchronization behaviors of two coupled fractional-order neuronal models and ring neuronal network under electromagnetic radiation are studied numerically. By research results, several novel phenomena and conclusions can be drawn. First, for the fractional-order HR model's ring neuronal network without electromagnetic radiation, if the fractional-order $q$ is changed, the threshold of the coupling strength when the network is in perfect synchronization will change. Furthermore, the change of fractional-order can induce the transition of periodic synchronization and chaotic synchronization. Second, for the two coupled neurons under electromagnetic radiation, the synchronization degree is influenced
\end{abstract}

Xin Yang $\cdot$ XueRen Li · Dong Wang

Aeronautics Engineering College,Air Force Engineering University

XiAn 710038, China

E-mail: y_angx_in@163.com

Dong Wang

E-mail: wangdong0120@126.com

XueRen Li

E-mail: lxr387625@sohu.com

GuangJun Zhang

Department of Basic Sciences,Air Force Engineering University

XiAn 710038, China

E-mail: zhanggj3@126.com by fractional-order and the feedback gain parameter $k_{1}$ . In addition, the fractional-order and parameter $k_{1}$ can induce the synchronization transition of bursting synchronization, perfect synchronization and phase synchronization. For the perfect synchronization, the synchronization transition of chaotic synchronization and periodic synchronization induced by $q$ and parameter $k_{1}$ is also observed. Especially, When the fractionalorder is small, like 0.6, the synchronization behavior will be more complex. Third, for the ring neuronal network under electromagnetic radiation, with the change of memory-conductance parameter $\beta$, parameter $k_{1}$ and fractional-order $q$ of electromagnetic radiation, the synchronization behaviors are different. When $\beta>0.02$, the synchronization will be strengthened with the decreasing of fractional-order. The parameter $k_{1}$ can induce the synchronization transition of perfect periodic10 synchronization, perfect periodic-7 synchronization, perfect periodic-5 synchronization and perfect periodic4 synchronization. It is hard for the system to synchronize and $q$ has little effect on the synchronization when $-0.06<\beta<0.02$. When $\beta<-0.06$, the network moves directly from asynchronization to perfect synchronization, and the synchronization factor goes from 0.1 to 1 with the small change of fractional-order. Larger the factional-order is, larger the range of synchronization is. The synchronization degree increases with the increasing of $k_{1}$.

Keywords Fractional-order · coupled neuronal network · electromagnetic radiation · synchronization transition 


\section{Introduction}

The firing behavior of neuron is a nonlinear process, and the neuron is a complex nonlinear dynamic system. In 1952, Hodgkin and Huxley used the equivalent circuits and large amounts of data from experiments to modeled and analyzed the data, then they constructed the Hodgkin-Huxley $(\mathrm{HH})$ neuron model through theoretical derivation[1]. Then, FItzHugu, Morris and Lecar, Hindmarsh and Rose proposed the FHN model[2], ML model[3], and HR model[4] respectively. Synchronization is an important phenomenon in neuronal system and operational mechanism of the brain. A number of researchers used the coupled neuronal models to try to explain some of the synchronization phenomena observed in the experiments.

For the synchronization behaviors of two coupled neurons and neuronal network, a lot of researches have been done by relevant scholars [5-27]. For the synchronization behaviors of two coupled neurons, the synchronization transition $[5,6]$ and the influence of different coupling way [7-9] are investigated. The synchronization transition of two coupled ML neurons is studied in [5], and it found that the bursting synchronization happens before spiking synchronization. The effects of memeristive synapse-coupled is investigated in [7]. When multiple neurons are coupled into a network, there will be more emergence phenomenon. Many researchers investigated the factors influencing network synchronization [10-23], such as the network topology [10-14], the time delay and partial time delay $[15,16]$, the coupled way and initial value [17-20], and the layers of networks [21]. In the previous researches, most neuron models are integer-order models. Fractional-order model can describe the real phenomenon more completely. The dynamic characteristics of the fractionalorder Hindmarsh-Rose (HR) neuronal model was investigated in [24], and it was found that different fractionalorder can induce different dynamic behaviors. For the fractional-order coupled neurons and neuronal networks, there are few studies. For the fractional-order coupled neurons, the two neurons reach the perfect synchronization through the design of the controller in most of the literature [25-27]. In [28], it is found that the change of fractional-order can change the synchronization mode. But the synchronization behaviors of fractional-order neuronal network aren't investigated.

Over the past period of time, the dynamic behaviors of neuron under electromagnetic radiation have been paid widely scholars' attention [26,29-31]. In fact, the neurons are usually exposed to electromagnetic radiation, and the external magnetic flux can affect the electromagnetic radiation of the neurons. In [30] and
[31], the external stimulus current and external electromagnetic radiation are omitted, respectively, and the corresponding models are proposed. According to [30], the single fractional-order neuron model under electromagnetic radiation is proposed, and the dynamic behaviors are investigated in [26]. The effects of electromagnetic radiation on the dynamical characteristic and synchronization behaviors of integer-order coupled neuronal networks were investigated in [31-34]. The synchronization behavior of one main network coupled with some sub-networks is investigated in [32]. In [33], it is found that the rhythm synchronization will happen under appropriate coupling strength and electromagnetic radiation. In [26] and [27], the two coupled neurons with and without time-delay were in perfect synchronization by designing appropriate controller, but they didn't investigate the effects of parameter and the synchronization transition. From the above analysis, the synchronization and synchronization transition of coupled neurons and neuronal network under electromagnetic radiation are not investigated in the previous researches. In the researches mentioned above, most method to study the fractional-order system are the predict-corrector method [35]. Predict-corrector method has a high accuracy, but the calculation is large. The ADM method [36] used in this paper has a higher accuracy, and calculation amount is smaller than that of the predict-corrector method [37].

From this perspective, this paper investigates the synchronization transition of coupled fractional-order neuronal networks. First, the ring network for fractionalorder HR model without electromagnetic radiation is constructed, and the synchronization transition induced by the fractional-order is investigated. Then, different from [25-27], the synchronization transition of two coupled neurons under electromagnetic radiation induced by the fractional-order, coupling strength and parameter $k_{1}$ is studied. Finally, this paper investigates the synchronization behavior of the ring neuronal network under electromagnetic radiation.

\section{Model description}

In this paper, in order to analyze the dynamic behaviors of the fractional-order neuronal network, the HR model is adopted for single neuron model. The HR model is described as follows [24]:

$$
\left\{\begin{array}{l}
D_{t}^{q} x=y-a x^{3}+b x^{2}-z+I \\
D_{t}^{q} y=c-d x^{2}-y \\
D_{t}^{q} z=r[s(x-\bar{x})-z]
\end{array}\right.
$$

Where $x$ is the membrane action potential, $y$ is a recovery variable, $z$ is a slow adaption current, $D_{t}^{q}$ is 
the differential operator defined by Caputo, $\mathrm{q}$ is the fractional-order. I is the external stimulus current. In this paper, parameters except fractional-order and external stimulus current are fixed as $a=1, b=3, c=$ $1, d=5, r=0.006, \bar{x}=-1.56, s=4$ [28]. The fractionalorder ring neuronal network without electromagnetic radiation can be described as follows:

$$
\left\{\begin{aligned}
& D_{t}^{q} x_{i}= y_{i}-a x_{i}^{3}+b x_{i}^{2}-z_{i}+I \\
& \quad+\frac{C}{2 P} \sum_{j=i-p}^{i+p} a_{i j}\left(x_{j}-x_{i}\right) \\
& D_{t}^{q} y_{i}=c-d x_{i}^{2}-y_{i} \\
& D_{t}^{q} z_{i}=r\left[s\left(x_{i}-\bar{x}\right)-z_{i}\right]
\end{aligned}\right.
$$

Where $i=1,2, \ldots, N, C$ is the coupling strength of the network. If node $\mathrm{i}$ and node $\mathrm{j}$ are connected, then $a_{i j}=a_{j i}=1$. Each neuron is symmetrically coupled to its $2 \mathrm{P}$ nearest neighbors. It is important to notice that $j=-m,(m=1,2, .$.$) implies node \mathrm{j}$ coupled with node $101-m$. In this paper, the parameter $\mathrm{P}$ is set as 1 . The network structure is shown in Fig.1.

In this paper, the two coupled fractional-order neurons under electromagnetic radiation can be described as follows [29]:

$$
\left\{\begin{aligned}
D_{t}^{q} x_{1,2}= & y_{1,2}-a x_{1,2}^{3}+b x_{1,2}^{2}-z_{1,2}+I \\
& +k_{1} W\left(\phi_{1,2}\right) x_{1,2}+C\left(x_{2,1}-x_{1,2}\right) \\
D_{t}^{q} y_{1,2}= & c-d x_{1,2}^{2}-y_{1,2} \\
D_{t}^{q} z_{1,2}= & r\left[s\left(x_{1,2}-\bar{x}\right)-z_{1,2}\right] \\
D_{t}^{q} \phi_{1,2}= & x_{1,2}-k_{2} \phi_{1,2}+\phi_{0}
\end{aligned}\right.
$$

$\phi$ denotes the magnetic flux across the cell membrance. $\phi_{0}$ is the external magnetic flux. $k_{1}$ is the feedback gain. The cubic flux-controlled memristor model $W(\phi)=$ $d q(\phi) / d \phi=\alpha+3 \beta \phi^{3}$ is introduced in this model, and it is used to estimate the effect of feedback regulation on menberance potential when magnetic flux is changed. The two parameters, $\alpha$ and $\beta$, describe the memoryconductance, and they will vary with the environment and their own conditions. In this paper, $\alpha=0.2, k_{2}=$ $0.4, \phi_{0}=1$.

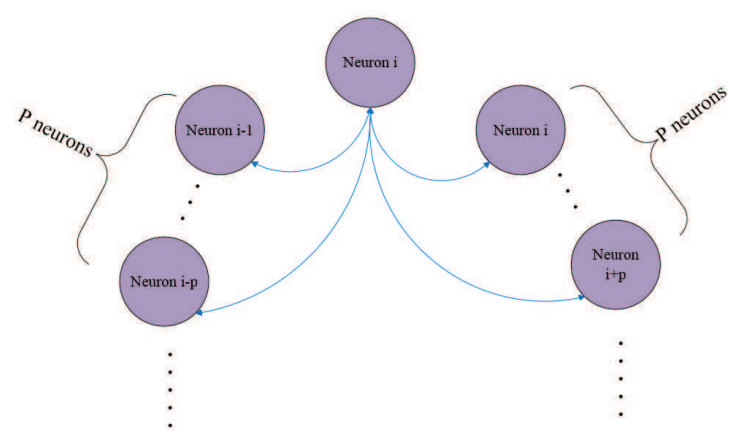

Fig. 1 The structure of neuronal network
The fractional-order neuronal ring network under electromagnetic radiation can be described as follows:

$$
\left\{\begin{aligned}
& D_{t}^{q} x_{i}= y_{i}-a x_{i}^{3}+b x_{i}^{2}-z_{i}+I+k_{1} W\left(\phi_{i}\right) x_{i} \\
& \quad+\frac{C}{2 P} \sum_{j=i-p}^{i+p} a_{i j}\left(x_{j}-x_{i}\right) \\
& D_{t}^{q} y_{i}=c-d x_{i}^{2}-y_{i} \\
& D_{t}^{q} z_{i}=r\left[s\left(x_{i}-\bar{x}\right)-z_{i}\right] \\
& D_{t}^{q} \phi_{i}=x_{i}-k_{2} \phi_{i}+\phi_{0}
\end{aligned}\right.
$$

\section{The synchronization behavior of fractional-order neuronal network without electromagnetic radiation}

In this paper, the synchronization factor $R$ is adopted to describe the synchronization of network. $R$ [34] is given by

$R=\frac{\left\langle F^{2}\right\rangle-\langle F\rangle^{2}}{\frac{1}{N} \sum_{i=1}^{N}\left(\left\langle x_{i}^{2}\right\rangle-\left\langle x_{i}\right\rangle^{2}\right)}, F=\frac{1}{N} \sum_{i=1}^{N} x_{i}$

where $\langle\cdot\rangle$ denotes the time averaging. The value of $R$ is between 0 and 1 , and it increases with decreasing average membrane potential errors. More precisely, perfect synchronization is expected when $\mathrm{R}$ is close to 1 and non-synchronization state may appear when $\mathrm{R}$ is close to 0 .

The ring network consists of 100 nodes. Numerical simulation is provided for the model (2) by utilizing the ADM method. As shown in Fig.2, the synchronous threshold of coupling strength will vary with the fractional-order. The change trend of threshold is to increase first and then decrease from Fig.2(b). In the previous researches, for the integer-order neuronal network, the threshold of coupling strength can change with other influence factors $[10,15]$, such as the structure of network and time-delay. In this paper, the fractionalorder is proved to be an influence factor.

As shown in previous researches, for the integerorder neuronal network, time-delay can induce the synchronization transition of neuronal network $[15,16]$. In this paper, the results will show that fractional-order not only makes the synchronous system become asynchronization, but also changes the synchronization mode. When the neuronal network is in perfect synchronization, Fig. 3 shows the phase diagram of $(z, x)$ when the fractional-order is $0.65,0.75,0.85$, and 0.95 . The neurons display periodic- 6 bursting, periodic-10 bursting, and periodic- 5 bursting when $q=0.65, q=0.75$, and $q=0.85$ as shown in Fig.3(a)(b)(c). The network is in perfect periodic-6, perfect periodic-10 and perfect periodic-5 synchronization. When $q=0.95$, as shown in Fig.3(d), neurons display chaotic bursting, so 


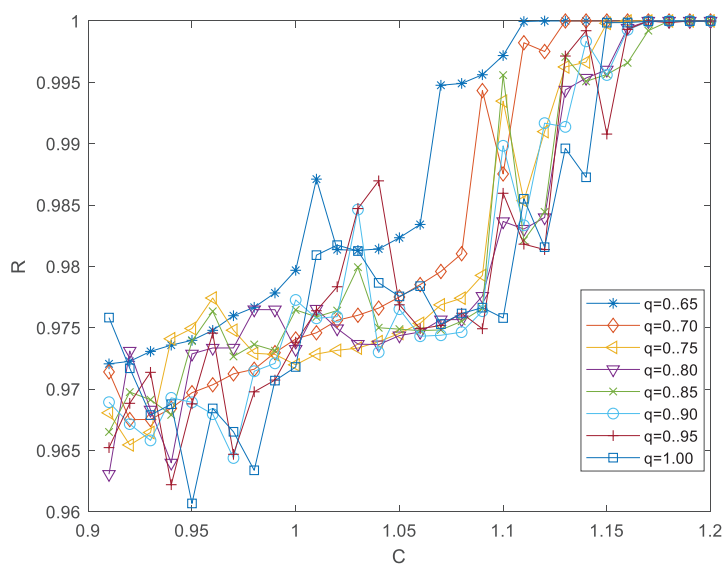

(a)

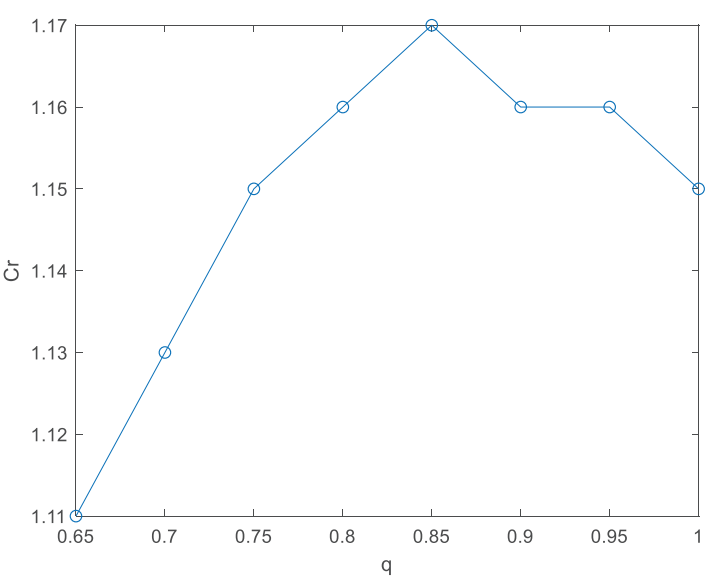

(b)

Fig. 2 (a)The curves of $R \sim C$ for different fractional-order. (b) the curve of $C_{r} \sim q$

the network is in perfect chaotic synchronization. The fractional-order can change the synchronization mode and induce the transition of perfect periodic synchronization and perfect chaotic synchronization.

\section{The synchronization behavior of two fractional-order coupled neurons under electromagnetic radiation}

The fractional-order HR neuron under electromagnetic radiation is investigated in [27], and two neurons reach perfect synchronization through adaptive control. But the synchronization behavior and synchronization transition induce by the fractional-order and other parameter are not reported in [27]. In this section, the synchronization behavior and synchronization transition induced by fractional-order and parameter $k_{1}$ are studied.

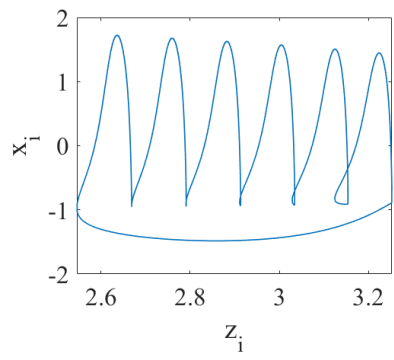

(a)

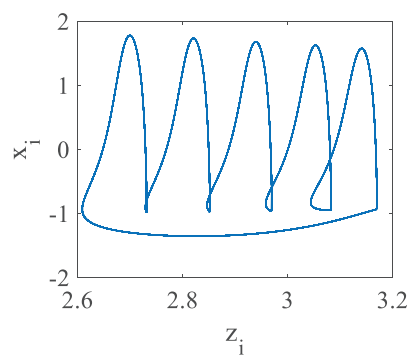

(c)

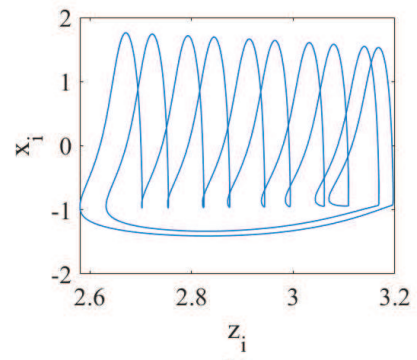

(b)

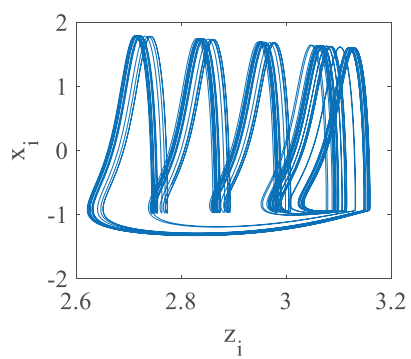

(d)
Fig. 3 the phase diagram of $(z, x)$ for (a) $q=0.65$, (b) $q=$ 0.75, (c) $q=0.85$, (d) $q=0.95$

In this part, the similarity function is introduced to measure the synchronization of the system [28]. The model of similarity function can be described as follows:

$S=\left[\frac{\left\langle\left(x_{1}(t)-x_{t}(t)\right)^{2}\right\rangle}{\left(\left\langle x_{1}^{2}(t)\right\rangle\left\langle x_{2}^{2}(t)\right\rangle\right)^{\frac{1}{2}}}\right]^{\frac{1}{2}}$

Where $\langle\cdot\rangle$ stands for the time average.

Obviously, the perfect synchronization can be observed when $S$ is equal to 0 , and bigger the $S$, worse the synchronization.

When the system is not in perfect synchronization, it may be in phase synchronization. The phase information can be calculated by detecting the time of sampled time series $\left(t_{1}, t_{2}, \ldots, t_{n}\right)$ across the Poincare section, and the phase is calculated by[39]

$\varphi=2 \pi \frac{t-t_{i}}{t_{i+1}-t_{i}}+2 \pi i, t_{i}<t<t_{i+1}$

And the phase difference between two neurons is defined by

$\Delta \varphi=\left|\varphi_{1}-\varphi_{2}\right|$

When the system is not in perfect and phase synchronization, it may be in bursting synchronization. Bursting synchronization means rhythm synchronization of slow variables, so bursting synchronization can be measured by the slow variable's similarity function[38]. 
The model of similarity function can be described as follows[38]:

$S_{z}=\left[\frac{\left\langle\left(z_{1}(t)-z_{t}(t)\right)^{2}\right\rangle}{\left(\left\langle z_{1}^{2}(t)\right\rangle\left\langle z_{2}^{2}(t)\right\rangle\right)^{\frac{1}{2}}}\right]^{\frac{1}{2}}$

4.1 The effects of fractional-order and coupling strength on the synchronization behaviors, and synchronization transition induced by the fractional-order under electromagnetic radiation

When $k_{1}=0.2$ fractional-order $q$ and parameter $C$ are varied in the regions $[0.55,1]$ and $[0.1,0.5]$, the similarity function is shown in Fig.4, where the blue color region with $\mathrm{S}$ equal to 0 denote the system in perfect synchronization. As shown in Fig.4, when $0.55<q<0.67$ and coupling strength around 0.367 , the similarity function undergoes a complex change, which means that synchronous threshold of coupling strength changes great. It is different from [28] because of the electromagnetic radiation. When $0.67<q<1$, the synchronous threshold of coupling strength decreases with increasing fractionalorder. When $0.366<C<0.378$, the $S \sim q$ curves are shown in Fig.5.

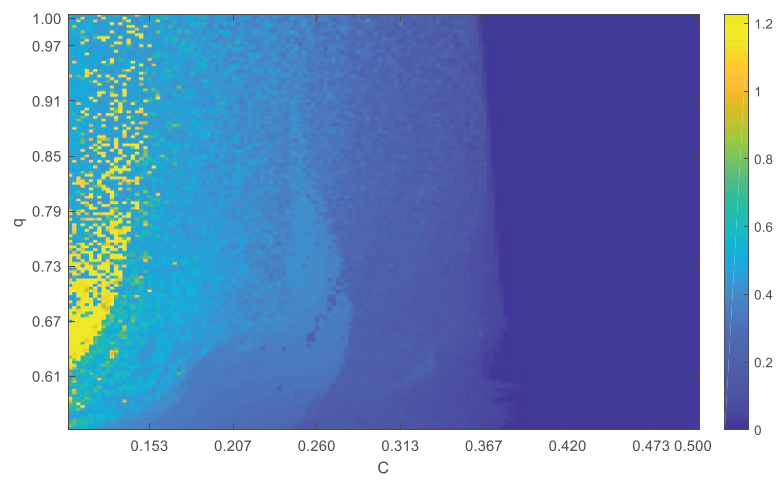

Fig. 4 Distribution for the similarity function in $q-c$ plane

As shown in Fig.4, the similarity function has striking changes when $0.366<C<0.378$, so the synchronization transition can be observed when $0.366<C<$ 0.378 . The synchronization transition when $C=0.372$ is taken as an example to observe the change of synchronization. When $q=0.56$, Fig.6(a) is the phase diagram of $\left(x_{1}, x_{2}\right)$, and Fig.6(b) is the corresponding time series of $x$, so the system is in imperfect synchronization. As shown in Fig.6(c)(f), the system is in perfect synchronization when $q=0.61$ and $q=0.9$, because the phase plane is located on a three quadrant angular bisector. The system is in imperfect synchronization when

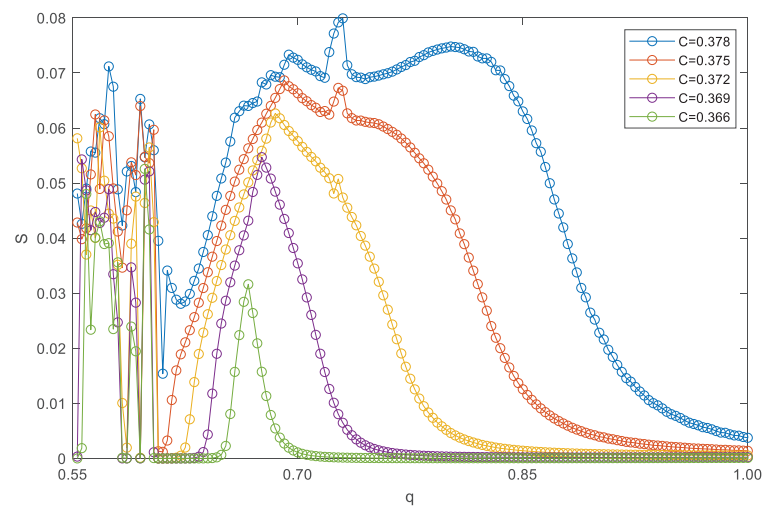

Fig. 5 The curves of $S \sim q$ for different coupling strength

$q=0.7$, shown as Fig.6(d) and Fig. 6(e), and the neurons display spiking.

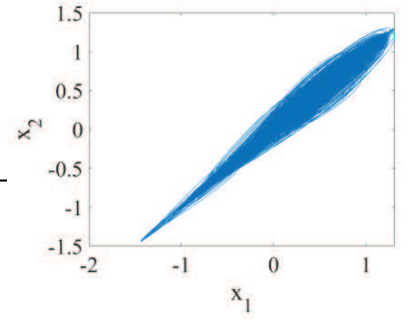

(a)

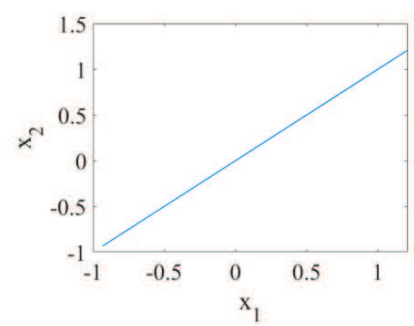

(c)

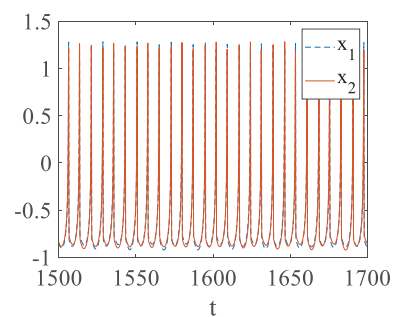

(e)

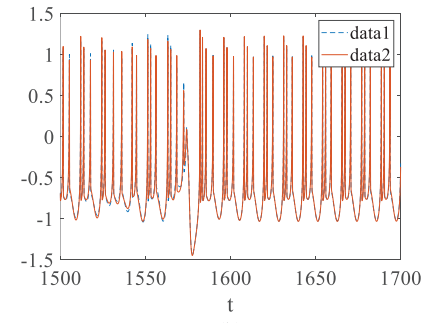

(b)

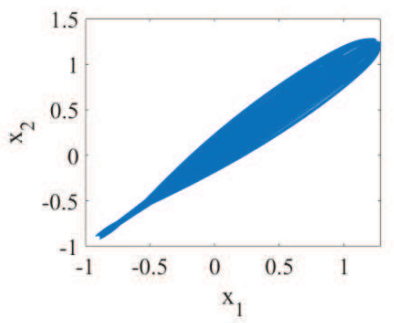

(d)

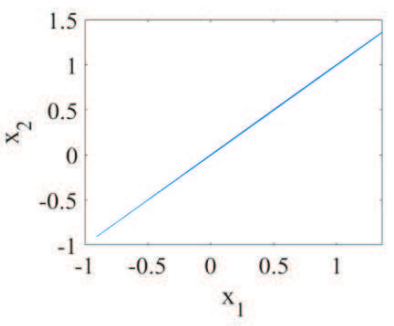

(f)
Fig. 6 The phase diagram of $\left(x_{1}, x_{2}\right)$ for (a) $q=0.56$, (c) $q=0.61$, (d) $q=0.7$, (f) $q=0.9$. And the corresponding time series of the $x$ for (b) $q=0.56$, (e) $q=0.7$

From Fig.6, the two neurons are in imperfect synchronization and the phase differences are calculated as follow. As show in Fig.7(a), the phase different are al- 
ways around 0 and $2 \pi$, so the system gets in phase synchronization. From the Fig.7(b), the phase difference is around 0 , so the system is also in phase synchronization when $q=0.7$.

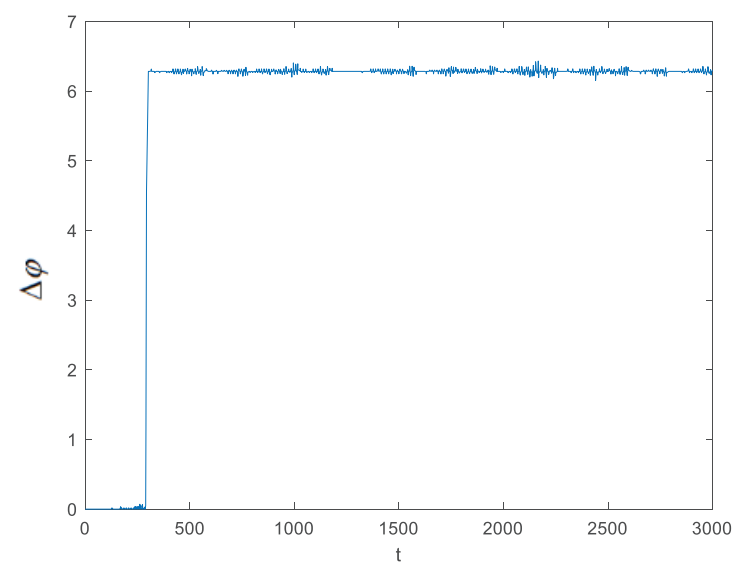

(a)

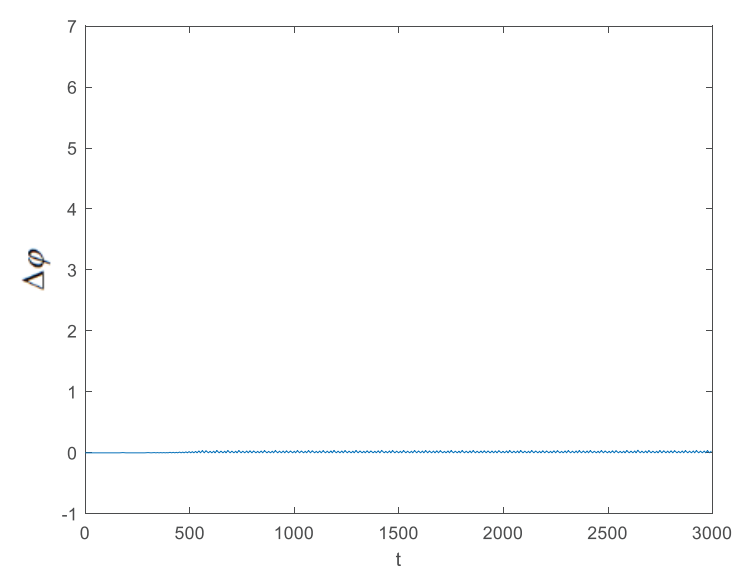

(b)

Fig. 7 The phase difference varied with time (a) $q=0.56$,(b) $q=0.7$

As the analysis mentioned above, the synchronization transition mode induced by fractional-order is phase synchronization - perfect synchronization - phase synchronization - perfect synchronization. The transition mode is more complex than the coupled fractional-order neurons without electromagnetic radiation [28].

As shown in Fig.4, the two neurons are in perfect synchronization when $C=0.5$ and $0.55<q<1$. Now, the ISI bifurcation of first neuron for $C=0.5$ is shown in Fig.8. There exists double period bifurcation when $q=0.66$ and $q=0.61$ with the decreas- ing of fractional-order. The system is in chaotic perfect synchronization when $0.57<q<0.59$, but the system is in periodic perfect synchronization at other fractional-order. The Fig.9 show the phase diagram of $\left(z_{1}, x_{1}\right)$ and $\left(z_{2}, x_{2}\right)$ when $q=0.56, q=0.58, q=0.6$, $q=0.65$ and $q=0.9$ for $C=0.5$. The neurons display periodic-3 bursting, chaotic bursting, periodic- 4 bursting, periodic-2 bursting, and spiking respectively. The synchronization transition, which is perfect periodic3 synchronization - perfect chaotic synchronization perfect periodic-4 synchronization - perfect periodic2 synchronization - perfect spiking synchronization, is observed. When the system is in perfect synchronization, the transition mode is also more complex than the coupled neurons without electromagnetic radiation[28], in which the synchronization mode is only periodic synchronization in all fractional-order [28]. The results can be concluded that when the system is in perfect synchronization, fractional-order and electromagnetic radiation can change the synchronization mode.

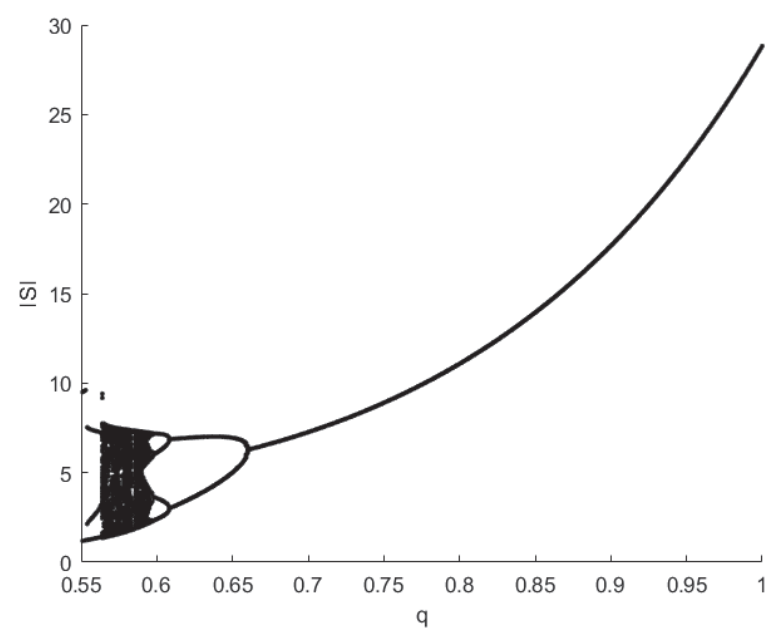

Fig. 8 The ISI bifurcation of first neuron

4.2 The effect of parameter $k_{1}$ and coupling strength on the synchronization, and synchronization transition induced by $k_{1}$ under different fractional-order

In this section, the influence of parameter $k_{1}$ and coupling strength on the synchronization behaviors, and the synchronization transition induced by $k_{1}$ will be investigated. The similarity function, phase difference, and slow variable's similarity function also are used to measure the synchronization degree of the system. Form the analysis mentioned above, different fractional-order will induce different dynamic behaviors. The influence 


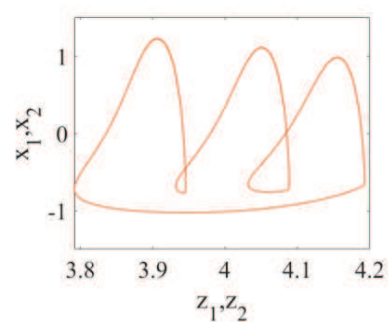

(a)

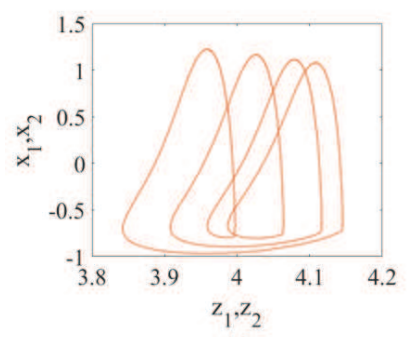

(c)

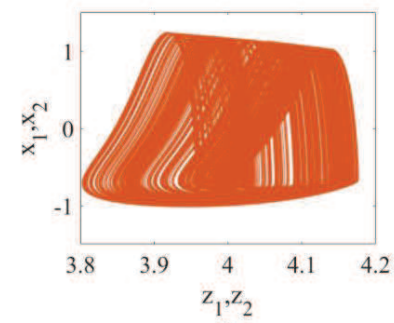

(b)

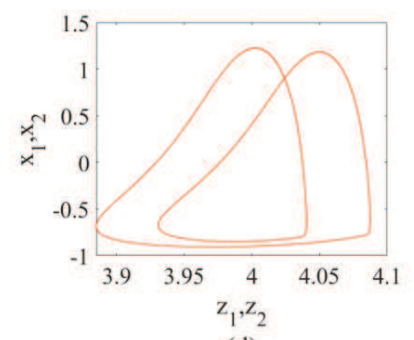

(d)

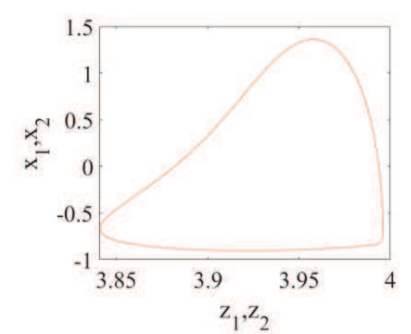

(e)

Fig. 9 The phase diagram of $\left(z_{1}, x_{1}\right)$ and $\left(z_{2}, x_{2}\right)$ for (a) $q=$ 0.56 , (b) $q=0.58$, (c) $q=0.6$, (d) $q=0.65$, (e) $q=0.9$

of parameter $k_{1}$ and coupling strength on synchronization will be studied under different fractional-order.

Therefore, the value of the similarity function in the plane of $\left(C, k_{1}\right)$ for several value of fractional-order is calculated, as shown in Fig.10. In Fig.10, the blue color region where $S$ equals 0 denote the system in perfect synchronization. Generally, the conclusion that large parameter $k_{1}$ and coupling strength can make the system in perfect synchronization can be drawn. But there is an exception, when $q=0.8$ and $q=0.95$. There are existing that the smaller $k_{1}$ can make the system be in perfect synchronization but the larger $k_{1}$ can't. As shown in Fig.11(a), when $q=0.8, C=0.34,0.1<$ $k_{1}<0.27$, the value of similarity function decreases with increasing of $k_{1}$, and then $S=0$ when $0.27<$ $k_{1}<0.35$. But with the increasing of $k_{1}$ sequentially, the $S>0$ when $0.35<k_{1}<0.39$. Then the $S=0$ when $k_{1}>0.39$. To observe the above process more visually, some figures about phase diagram and the corresponding time series are shown in Fig.11(b)-(f) when $q=0.8, C=0.34$. As shown in Fig.11(b)(c), the phase diagram of $\left(x_{1}, x_{2}\right)$ and the corresponding time
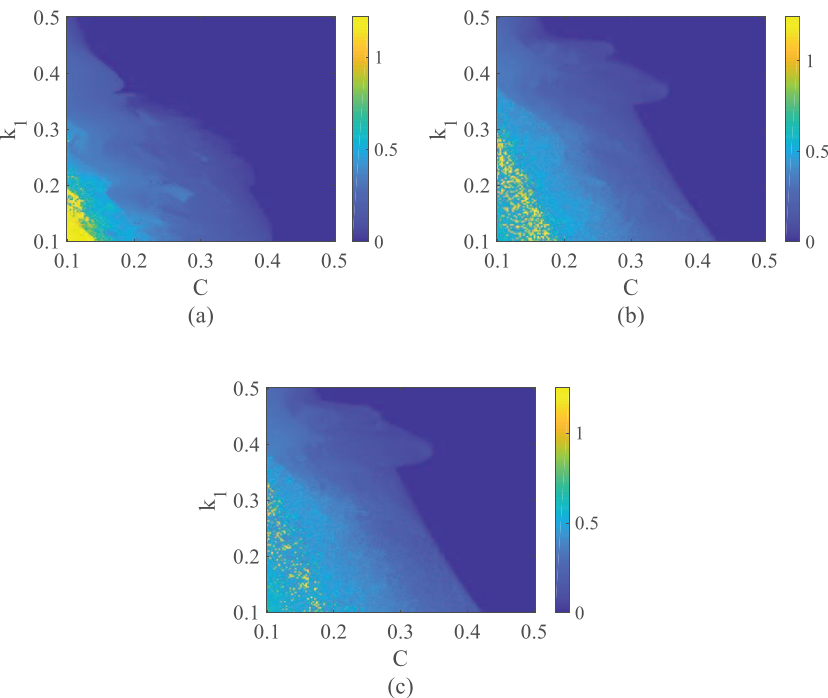

Fig. 10 Distribution for the similarity function in $k_{1}-C$ plane for (a) $q=0.6$, (b) $q=0.8$, (c) $q=0.95$

series of $x_{1}$ and $x_{2}$ show that the system is in imperfect synchronization when $k_{1}=0.2$. From Fig. 10(d)(f), the system is in perfect synchronization when $k_{1}=0.3$ and $k_{1}=0.4$. The system is in asynchronization when $k_{1}=0.37$, as shown in Fig.11(e).

The phase differences of $x_{1}$ and $x_{2}$ when $k_{1}=0.2$ and $k_{1}=0.37$ are plot in Fig.12. we can conclude that the two neurons are in phase synchronization when $k_{1}=0.37$, because the phase different is small around 0 . The two neurons are not in phase synchronization when $k_{1}=0.2$ but the phase is locked in some time periods, and the slow variable's similarity function is calculated as $S_{z}=0.05$, so the two neurons are in bursting synchronization. The synchronization transition induced by parameter $k_{1}$, that is bursting synchronization - perfect synchronization - phase synchronization - perfect synchronization can be observed.

Meanwhile Fig.10 also shows that the range of synchronization when $q=0.6$ is largest in three value of $q$. As shown in Fig.13, $C=0.26, k_{1}=0.38$, the system is in asynchronization when $q=0.8$ and $q=0.95$, but system is in perfect synchronization when $q=0.6$.

If we change the parameter $k_{1}$ when the system is in perfect synchronization $(C=0.5)$ for several value of $q$, the synchronization mode will change. The ISI bifurcation of first neuron is plot in Fig.14. As shown in Fig.14(a), when $0.1<k_{1}<0.14$ and $0.25<k_{1}<0.33$, the neurons display chaotic firing, but when $0.14<$ $k_{1}<0.24$ and $k_{1}>0.33$, the neurons display periodic firing. When $q=0.6, C=0.5$, the parameter $k_{1}$ will induce the transition of chaotic perfect synchronization and periodic perfect synchronization. As shown in Fig.14(b), when $q=0.8, C=0.5$. There is double pe- 


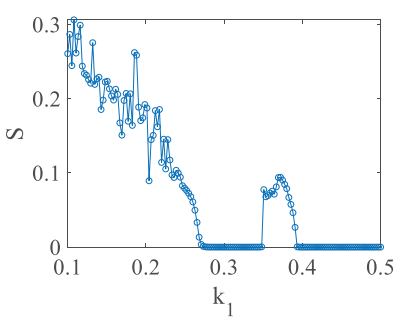

(a)

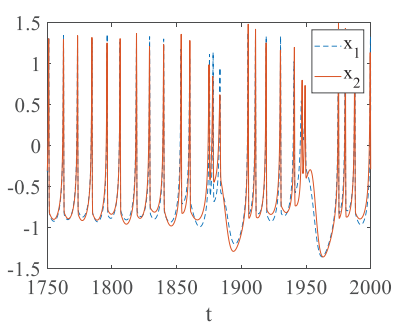

(c)

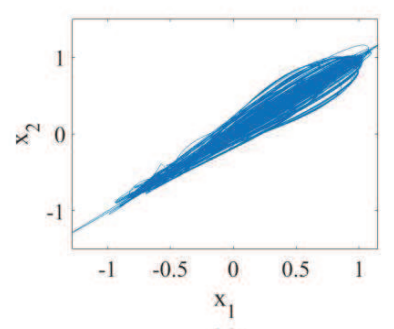

(e)

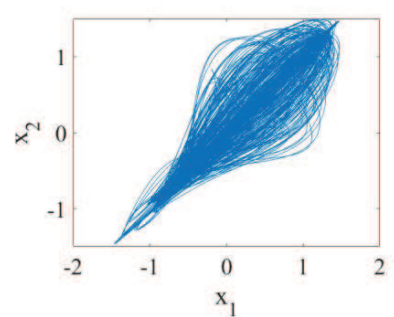

(b)

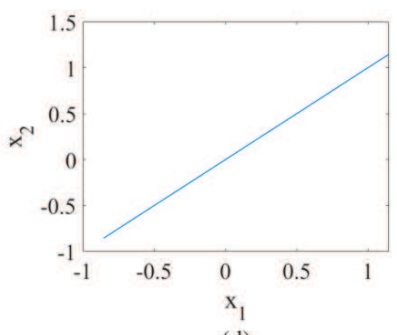

(d)

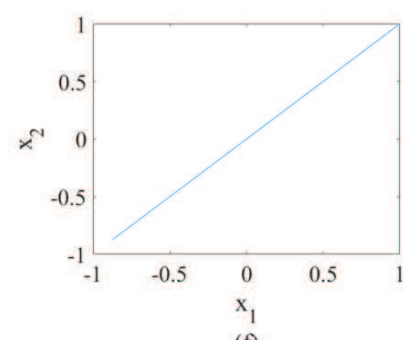

(f)

Fig. 11 (a)The curve of $S \sim k_{1}$ for $C=0.34, q=0.8$. The phase diagram of $\left(x_{1}, x_{2}\right)$ for (b) $k_{1}=0.2$, (d) $k_{1}=0.3$, (e) $k_{1}=0.37$, (f) $k_{1}=0.4$. (c) the corresponding time series of the $x$ for $k_{1}=0.37$

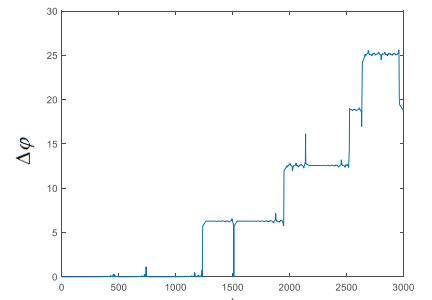

(a)

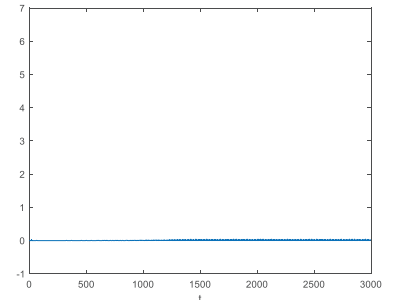

(b)

Fig. 12 The phase difference varied with time (a) $k_{1}=0.2,(\mathrm{~b})$ $k_{1}=0.37$

riod bifurcation when $k_{1}=0.38 . k_{1}$ also can induce the synchronization transition. Thus, the other kind of synchronization transition, which firing mode of two perfect synchronization neurons are different, induced by $k_{1}$ is also observed.

The phase diagrams of $\left(z_{1}, x_{1}\right)$ and $\left(z_{2}, x_{2}\right)$ when $C=0.5$ are shown in Fig. 15 . And the synchronization transition induced by the parameter $k_{1}$ can be explained. Fig.15(a)(c) shows that the neurons display

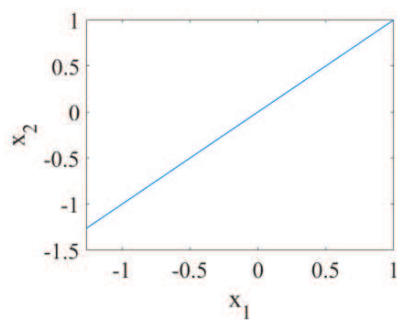

(a)

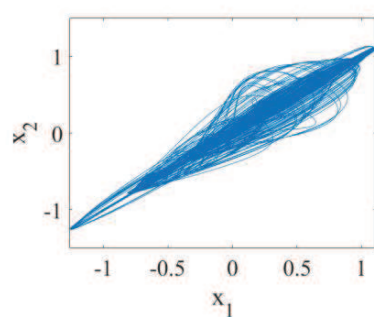

(b)

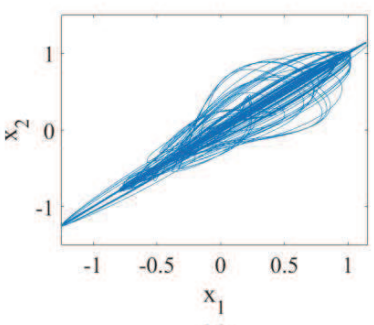

(c)

Fig. 13 The phase diagram of $\left(x_{1}, x_{2}\right)$ for (a) $q=0.6$, (b) $q=0.8$, (c) $q=0.95$

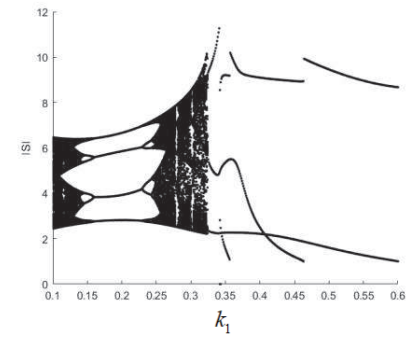

(a)

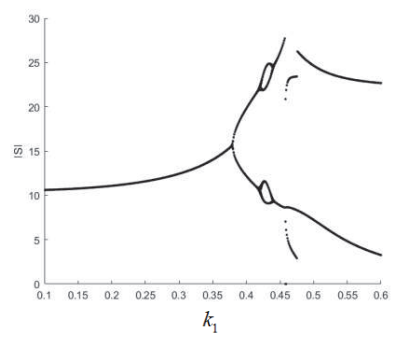

(b)
Fig. 14 The ISI bifurcation of first neuron for $C=0.5$ (a) $q=0.6,(\mathrm{~b}) q=0.8$

chaotic firing, so the system is in chaotic synchronization when $q=0.6, k_{1}=0.12$ and $q=0.6, k_{1}=0.3$. Form Fig.15(b)(d) The neurons display periodic-4 bursting and periodic- 3 bursting, so the system is in periodic synchronization when $q=0.6, k_{1}=0.16$ and $q=0.6, k_{1}=0.35$. The system is in periodic synchronization at all value of $k_{1}$ when $q=0.8$, but the neurons display spiking firing and periodic-2 bursting when $k_{1}=0.15$ and $k_{1}=0.4$ as shown in Fig.15(e)(f).

In [26], the effects of parameter $k_{1}$ on the synchronization are not investigated. And in [33], when the neuron model is integer-order model, the synchronization mode is perfect spiking synchronization for all $k_{1}$ when $C=0.5$. The conclusion can be summarized that parameter $k_{1}$ can induce the synchronization transition and the synchronization behaviors are different when fractional-order varies. As shown in Fig.14 and Fig.15, the small fractional-order, such as 0.6 , can induce more complex synchronization transition. 


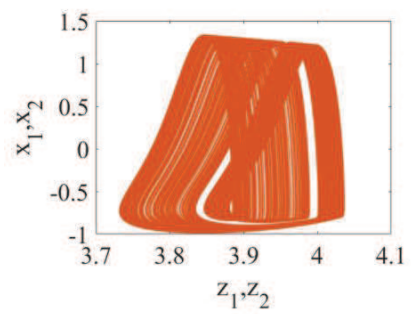

(a)

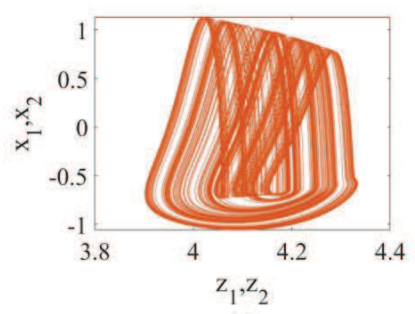

(c)

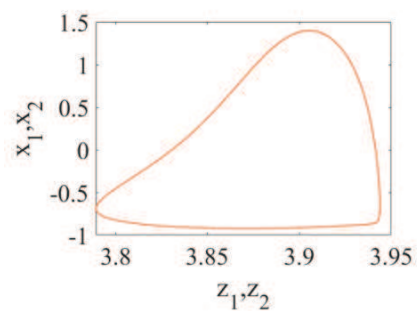

(e)

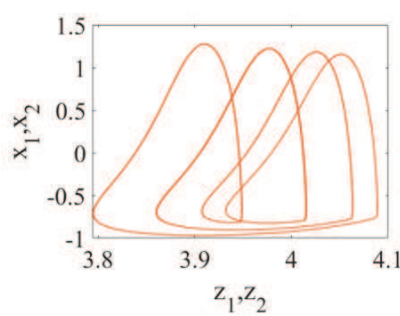

(b)

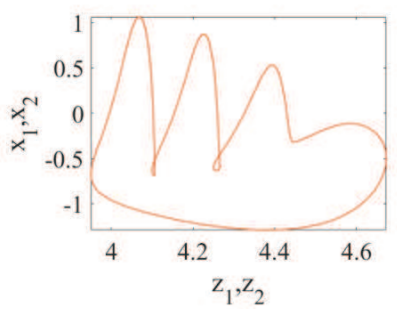

(d)

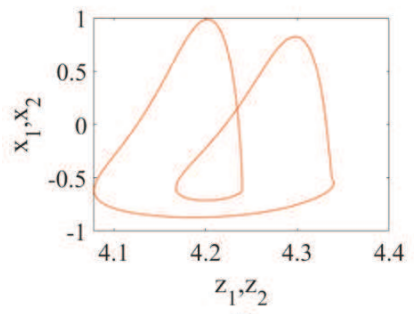

(f)
Fig. 15 The phase diagram of $\left(z_{1}, x_{1}\right)$ and $\left(z_{2}, x_{2}\right)$ for $C=$ 0.5 (a) $q=0.6, k_{1}=0.12$, (b) $q=0.6, k_{1}=0.16$, (c) $q=$ $0.6, k_{1}=0.3$, (d) $q=0.6, k_{1}=0.35$, (e) $q=0.8, k_{1}=0.15$, (f) $q=0.8, k_{1}=0.4$

\section{The synchronization behavior of fractional-order neuronal network under electromagnetic radiation}

In this part, the effects of some parameters on synchronization behaviors of ring neuronal network under electromagnetic radiation will be investigated. In [33], the integer-order neuron's firing activity is influenced by the parameter $k_{1}$. In this paper, the firing activity is also influenced by the parameter $\beta$ except $k_{1}$. In addition, in this paper, the results show that fractionalorder cannot influence the neurons' firing mode in the network. It is obviously different with the two coupled neurons under electromagnetic radiation, single neuron and the ring neuronal network without electromagnetic radiation. Although the fractional-order can't influence the neuronal firing mode, the fractional-order can influence the synchronization behaviors of the ring neuronal network under electromagnetic radiation at different conditions. For example, the ring neuronal network constructed by integer-order neuronal models is in perfect synchronization, but the perfect synchronization maybe destroyed in the same conditions when the integer-order neuronal models is instead of fractional-order neuronal models, vice versa. Therefore, this part focus on the effects of parameter $\beta, k_{1}$ and $q$ on the synchronization behaviors and synchronization transition of ring neuronal network constructed by fractional-order neuronal models in the next.

Now the firing mode with different parameter $\beta$ when $k_{1}=0.4, C=10$ will be plot in Fig.16, and the other parameters are set as mentioned in section 1. Fig.16 shows the firing mode of the first neuron when the parameter $\beta$ is different. From numerical simulation, we can find that the neurons display chaotic bursting, spiking firing, and periodic bursting when $-0.06<$ $\beta<0.02, \beta<-0.06$, and $\beta>0.02$. In this section, the synchronization behaviors in the three regions of $\beta$ will be studied numerically.

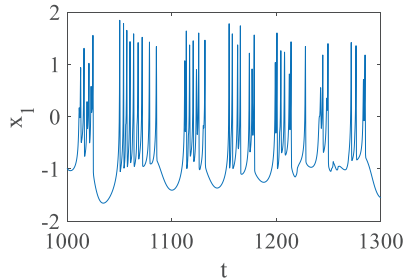

(a)

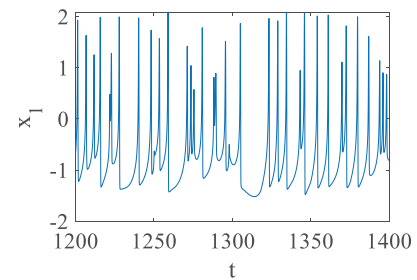

(b)

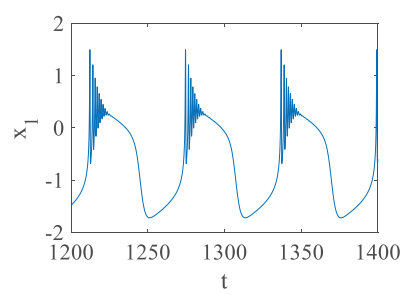

(c)

Fig. 16 The corresponding time series of $x$ for $q=0.8$ (a) $\beta=-0.02$, (b) $\beta=-0.08$, (c) $\beta=0.04$

The Bursting synchronization of neuronal network can be described by slow variable's synchronization factor $R_{z} \cdot R_{z}$ is given by

$R_{z}=\frac{\left\langle F_{z}^{2}\right\rangle-\left\langle F_{z}\right\rangle^{2}}{\frac{1}{N} \sum_{i=1}^{N}\left(\left\langle z_{i}^{2}\right\rangle-\left\langle z_{i}\right\rangle^{2}\right)}, F_{z}=\frac{1}{N} \sum_{i=1}^{N} z_{i}$

where $\langle\cdot\rangle$ denotes the time averaging. When $R_{z}$ is close to 1 , the system reaches bursting synchronization.

5.1 the synchronization behavior of neuronal network when $\beta>0.02$

In this part, $\beta=0.04$ is set as an example. The curve of $R \sim C$ for different $k_{1}$ are shown in Fig.17. From 
Fig.17, we will find that the effect of parameter $k_{1}$ and coupling strength on synchronization behaviors. The synchronization factor increases with the increase of coupling strength and $k_{1}$. When the $\beta=0.04, k_{1}$ plays a dominant role in network synchronization, because the network can't reach perfect synchronization when $k_{1}=0.15,0.2,0.25,0.3$ and the coupling strength ranges from 1 to 16 . The state of network can be transformed from asynchronization to perfect synchronization with minor change of $k_{1}$ when $k_{1}>0.35$.

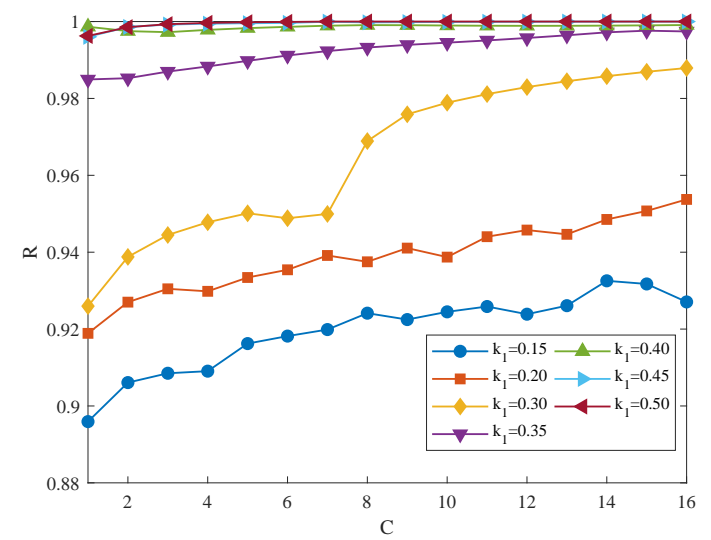

Fig. 17 The curves of $R \sim C$ for different $k_{1}$

To observe the effect of fractional-order, $k_{1}$ is set as 0.4. Fractional-order cannot influence the neurons' firing mode, but it can change the synchronization degree. Fig. 18 shows the $R \sim q$ curves for different coupling strength. We can conclude that the increase of fractional-order will weaken the synchronization of the neuronal network. The synchronization is robust against alterations of coupling strength when fractional-order is small. When the fractional-order is 0.7 , the difference of synchronization factor between coupling strength 1 and 6 is only 0.05 , but when the fractional-order is 1 , the difference becomes 0.2 .

Fig.19 shows neuronal network's spatiotemporal patterns and corresponding snapshots when $C=4.5, k_{1}=$ 0.35 at $t=2218$. It is concluded that the strength of synchronization is higher when the fractional-order is 0.7 than the one when the fractional-order is 0.98 . When $C=4.5, q=0.7$, the $x_{i}$ are uniform distributed value, so the network is in nearly perfect synchronization. When $C=4.5, q=0.98$, the $x_{i}$ are disorderly. But the slow variable's synchronization factor $R_{z}$ is calculated as 0.99 , so the network is in bursting synchronization. The fractional-order can also induce the transition of perfect synchronization and bursting synchronization when the ring network under electromagnetic radiation.

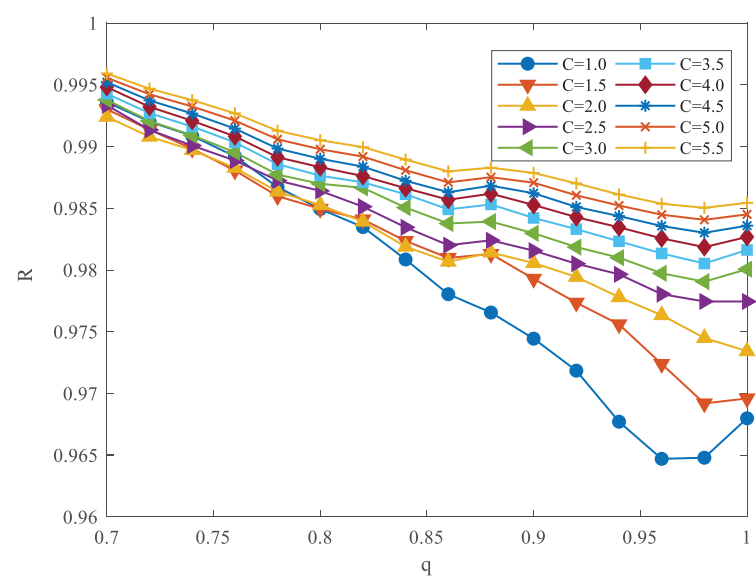

Fig. 18 The curves of $R \sim q$ for different coupling strength

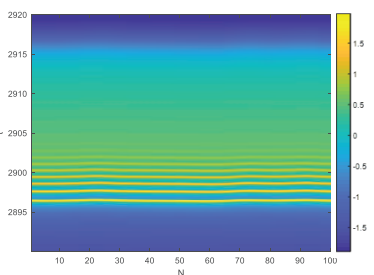

(a)

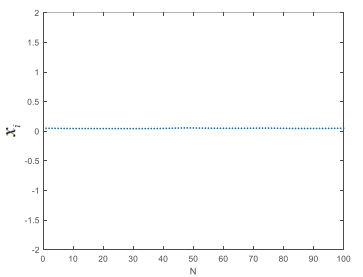

(c)

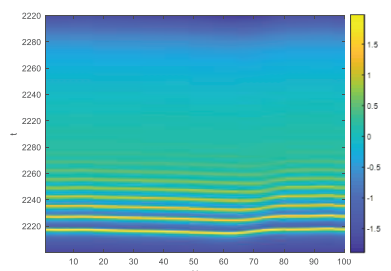

(b)

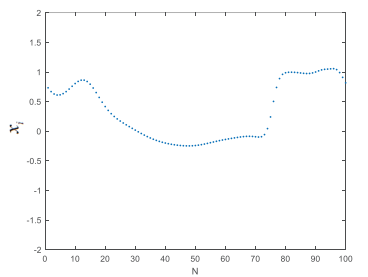

(d)
Fig. 19 The neuronal network's spatiotemporal patterns for (a) $q=0.7$, (b) $q=0.98$. And the corresponding snapshots at $t=2218 s$ for $(\mathrm{c}) q=0.7$, (d) $q=0.98$

In addition, $k_{1}$ can also induce synchronization transition, that is to say, the firing mode of neurons will change for different $k_{1}$. The parameters are set as $C=$ $10, q=0.8$. Fig.20 show the phase diagram of $\left(z_{i}, x_{i}\right)$ when $k_{1}=0.4,0.5,0.6,0.7$. As shown in Fig.20, the neurons of the network display periodic-10 bursting, periodic- 7 bursting, periodic- 5 bursting, and periodic4 bursting when $k_{1}=0.4,0.5,0.6,0.7$. The number of spiking in one bursting decreases with the increasing of $k_{1}$, so the synchronization mode varies with $k_{1}$. The synchronization transition induced by $k_{1}$, which is perfect periodic-10 synchronization - perfect periodic-7 synchronization - perfect periodic-5 synchronization perfect periodic-4 synchronization, is observed. 


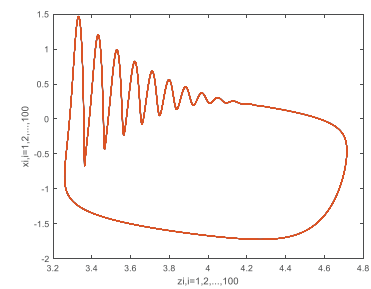

(a)

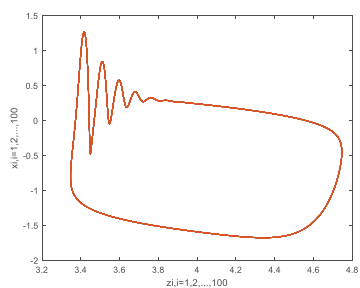

(c)

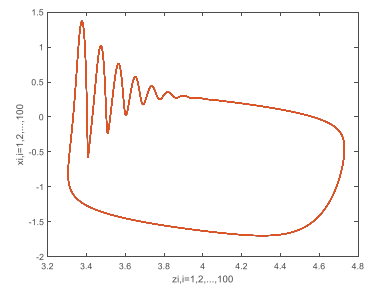

(b)

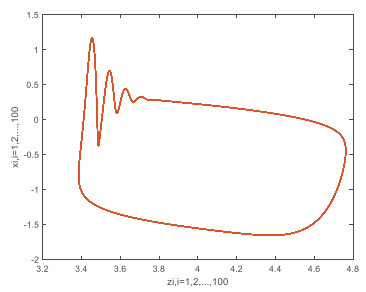

(d)
Fig. 20 The phase diagram of $\left(z_{i}, x_{i}\right)$ for (a) $k_{1}=0.4$, (b) $k_{1}=0.5$, (c) $k_{1}=0.6$, (d) $k_{1}=0.7$

5.2 the synchronization behavior of neuronal network when $-0.06<\beta<0.02$

In this part, $\beta=0.02$ is set as an example. The synchronization behavior is different from the above analysis. As shown in Fig.21, the synchronization factor also increases with the increase of coupling strength and $K_{1}$, but we can conclude that it is difficult to reach synchronization. The coupling strength ranges from 1 to 16 , the synchronization factor just reaches around 0.3 .

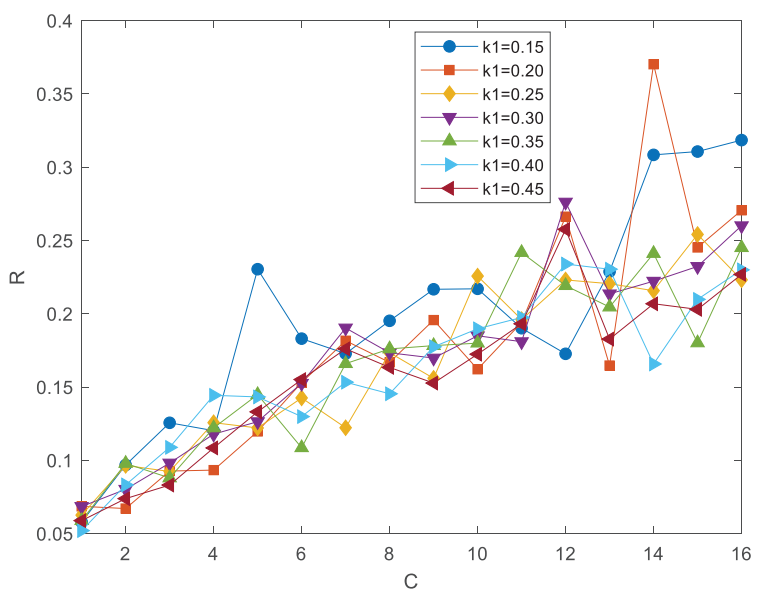

Fig. 21 The curves of $R \sim C$ for different $k_{1}$

Neuronal network's spatiotemporal patterns and corresponding snapshots at $t=2500$ when $K_{1}=0.4$ are shown in Fig.22. It is found that the $x_{i 1}$ are disorderly when the coupling strength is 1 and 16 . And the degree of synchronization is higher when the coupling strength is 16 than the one when the coupling strength is 1 , but they are all not in perfect synchronization. After calculating the slow variable's synchronization factor, the network is not in bursting synchronization. This part of view is different from the case of network without electromagnetic radiation, as shown in Fig.3(d), under the same fractional-order and coupling strength, the network can be in perfect synchronization. In this condition, it is necessary to mention that the synchronization behaviors are minimally affected by fractional-order.

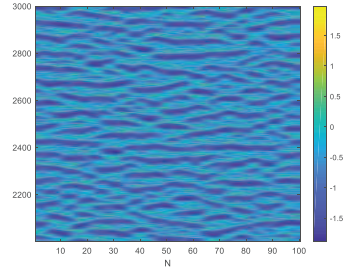

(a)

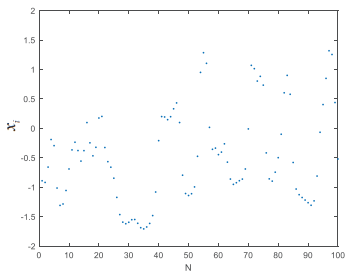

(c)

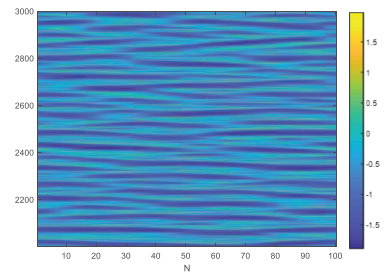

(b)

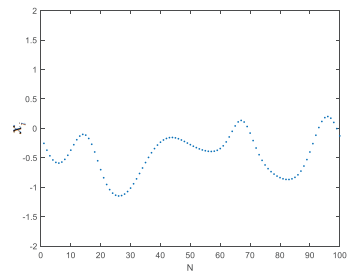

(d)
Fig. 22 The neuronal network's spatiotemporal patterns for $k_{1}=0.4$ (a) $C=1$, (b) $C=16$. And the corresponding snapshots at $t=2500 \mathrm{~s}$ for $k_{1}=0.4$ (c) $C=1$, (d) $C=16$

5.3 the synchronization behavior of neuronal network when $\beta<-0.06$

In this part, $\beta=-0.08$ is set as an example. There will be some novel phenomenon. The $R \sim C$ curves for different fractional-order are plot in Fig.23. We can find that network is in perfect synchronization when $C>7$, but the neurons' dynamic behaviors are different when $6.4<C<7$ for different fractional-order. In addition, it can be found that the transition of synchronization and asynchronization is abrupt when the fractional-order and coupling strength changes slightly. The synchronization factor changes 0.1 to 1 directly. This phenomenon is not found in previous studies.

Fig.24 shows the neuronal network's spatiotemporal patterns at different fractional-order and coupling strength. As shown in Fig.24, for each fractional-order, network will undergo several sudden transitions of asynchronization and perfect synchronization with the variation of coupling strength. The dynamic behavior of neurons in network is different at different fractionalorder and coupling strength. When $C=6.7$, network 


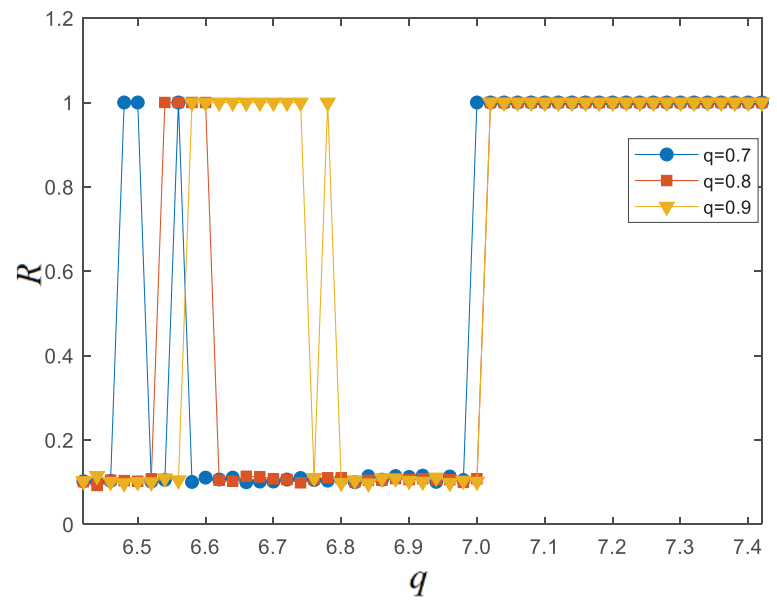

Fig. 23 The curves of $R \sim C$ for different $q$

is in asynchronization at $q=0.7$ and $q=0.8$, but network is in perfect synchronization at $q=0.9$. When $C=0.648$, network is in perfect synchronization at $q=0.7$, but network is in asynchronization at $q=0.8$ and $q=0.9$. When $\beta<-0.06$, fractional-order can also induce synchronization transition. And we can find that larger the factional-order is, larger the range of asynchronization from Fig.23. From the value of slow variable's synchronization factor when the network is not in perfect synchronization, the network cannot get in bursting synchronization. In the neuronal network without electromagnetic radiation, we cannot find the abrupt change of synchronization factor and synchronization behaviors.

For the influence of $k_{1}$, as shown in Fig.25, the synchronization degree increases when the $k_{1}$ increases, but the network is hard to get in perfect synchronization. The parameters are set as $q=0.8, C=6.7$.

\section{Conclusion}

This paper investigates the synchronization behavior and synchronization transition in three cases, which are the fractional-order HR model's ring neuronal network without electromagnetic radiation, two coupled fractional-order neuronal models under electromagnetic radiation and fractional-order ring neuronal network under electromagnetic radiation. First, for the fractionalorder model's ring neuronal network without electromagnetic radiation, like other influence factors reported in [21-33], the fractional-order can also change the threshold of the coupling strength. And the transition of periodic synchronization and chaotic synchronization induced by the fractional-order is found. Second, For the two coupled fractional-order neurons under electromagnetic radiation, the synchronous threshold of coupling strength will vary with the fractional-order. Compared with [28] (without electromagnetic radiation), the variation is more complex. The synchronization transition is also investigated in this situation. The transition of bursting synchronization, perfect synchronization, phase synchronization and perfect spiking synchronization is founded when the fractional-order changes. In addition, compared with [28], when the two coupled neurons are in perfect synchronization, the transition of perfect chaotic synchronization and perfect periodic synchronization will be founded when changing the fractionalorder and parameter $k_{1}$. And when the fractional-order is small like $q=0.6$, from the ISI bifurcation, the dynamics behavior will be more complex. Third, For the fractional-order ring neuronal network under electromagnetic radiation, it had been investigated that the parameter $k_{1}$ can change the firing activity in [33]. Different from the results in [33], this paper focus on the influence of parameter $\beta, k_{1}$ and fractional-order $q$ on synchronization behaviors and synchronization transition. In network, fractional-order cannot change the firing activity of neurons. It is obviously different with the two coupled neurons and neuronal network without electromagnetic radiation. When $\beta>0.02$, the synchronization degree decreases with fractional-order increasing. The parameter $k_{1}$ can induce the synchronization transition of perfect periodic-10 synchronization, perfect periodic-7 synchronization, perfect periodic- 5 synchronization and perfect periodic- 4 synchronization. When $-0.06<\beta<0.02$, the network is difficult to get into perfect synchronization, and the fractional-order is hard to change the synchronization degree. In particular, when $\beta<-0.06$, the network has sudden transition of asynchronization and perfect synchronization. The synchronization factor goes suddeny from 0.1 to 1 . And larger the factional-order is, larger the range of asynchronization is. The synchronization degree increases with the increasing of $k_{1}$.

Funding: No funding was received for conducting this study

Compliance with ethical standards Informed consent.

Conflict of Interest: The authors declare that they have no conflict of interest.

Availability of data and material: The datasets generated during and analysed during the current study are available from the corresponding author on reasonable request. 


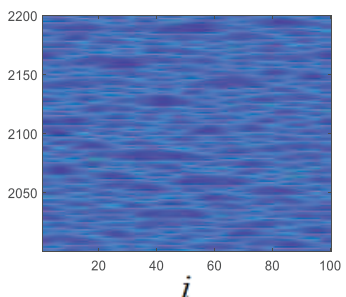

(a)

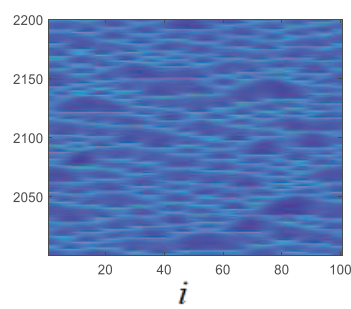

(e)

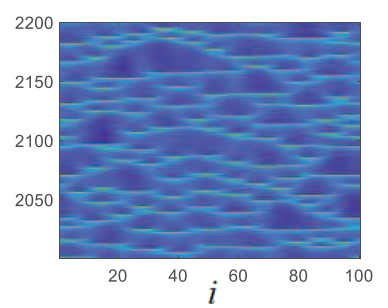

(i)

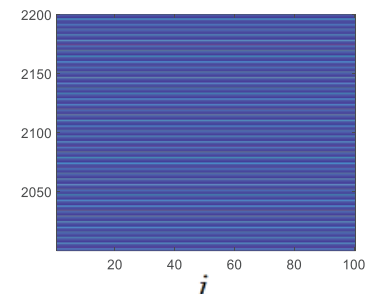

(b)

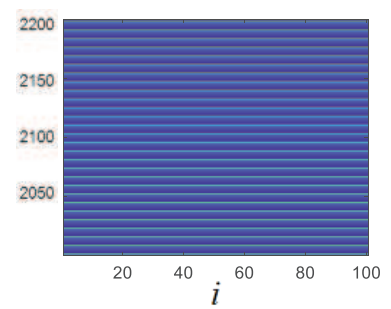

(f)

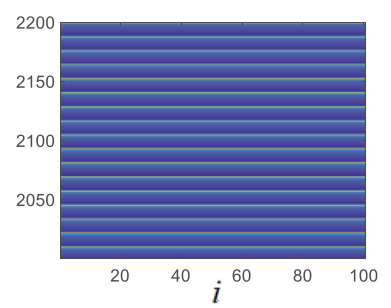

(j)

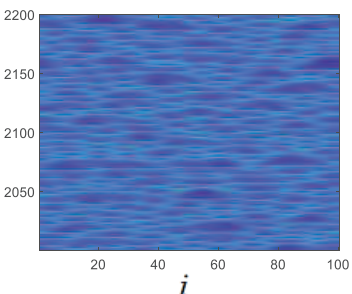

(c)

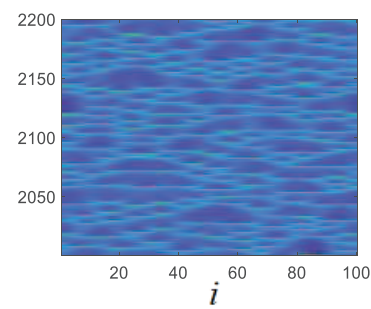

(g)

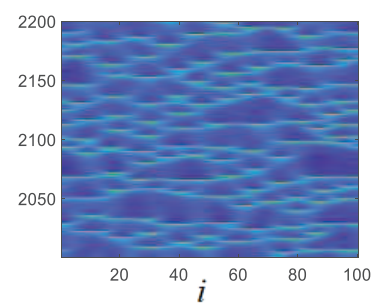

(k)

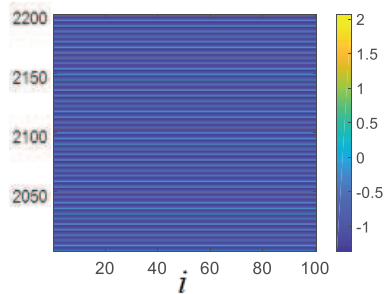

(d)

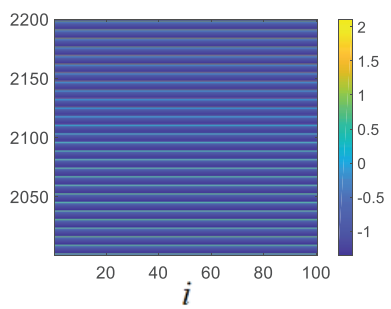

(h)

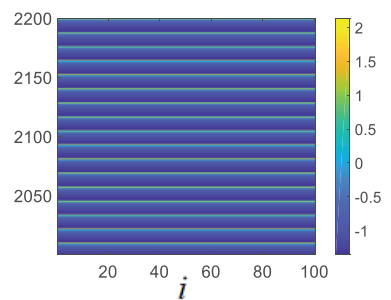

(1)

Fig. 24 The neuronal network's spatiotemporal patterns and corresponding snapshots for (a) $q=0.7, C=6.43$ (b) $q=0.7, C=$ $6.48(\mathrm{c}) q=0.7, C=6.7$ (d) $q=0.7, C=7.1(\mathrm{e}) q=0.8, C=6.48(\mathrm{f}) q=0.8, C=6.6(\mathrm{~g}) q=0.8, C=6.7(\mathrm{~h}) q=0.8, C=7.1(\mathrm{i})$ $q=0.9, C=6.48(\mathrm{j}) q=0.9, C=6.7(\mathrm{k}) q=0.9, C=6.9(\mathrm{l}) q=0.9, C=7.1$

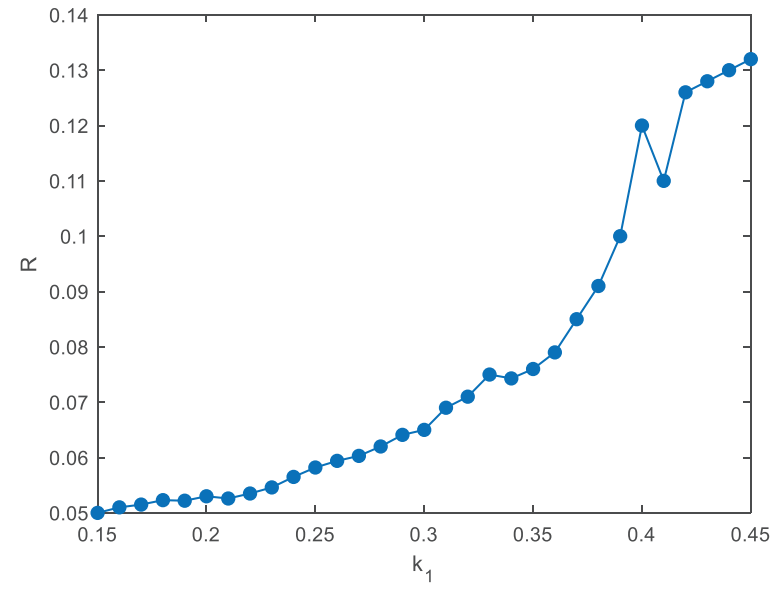

Fig. 25 The $R \sim k_{1}$ for $q=0.8$ and $C=6.7$

Author contributions: Material preparation, data collection and analysis were performed by [Xin Yang], [GuangJu Zhang]. The first draft of the manuscript was written by [Xin Yang] and all authors commented on previous ver- sions of the manuscript. All authors read and approved the final manuscript.

\section{References}

1. Hodgkin, A.L., Huxley, A.F, A quantitative description of membrane current and its application to conduction and excitation in nerve, J. Physiol. 117(4), 500-544 (1952)

2. FitzHugh, R, Impulses and physiological states in theoretical models of nerve membrane, Biophys. J. 1(6), 445-466 (1961)

3. Morris, C., Lecar, H., Voltage oscillations in the barnacle giant muscle fiber, Biophys. J., 35(1), 193-213 (1981)

4. Hindmarsh, J.L., Rose, R., A model of neuronal bursting using three coupled first order differential equations, Soc. Lond. B Biol., 221(1222), 87-102 (1984)

5. Wang QY, Lu QS, and Wang HX, Transition to complete synchronization via near-synchronization in two coupled chaotic neurons, Chinese Phys., 14, 2189-2195 (2005)

6. Wang QY,Duan ZS, Feng ZS,Chen GR,Lu QS, Synchronization transition in gap-junction-coupled leech neurons, Physica A, 387(16) (2008)

7. Xu F, Zhang J, Fang T, et al, Synchronous dynamics in neural system coupled with memristive synapse, Nonlinear Dynam., (2008)

8. Jun Ma,Lv Mi,Ping Zhou,Ying Xu,Tasawar Hayat, Phase synchronization between two neurons induced by coupling of electromagnetic field, Appl. Math. Comput.,30 (2017) 
9. Fatemeh Parastesh,Hamed Azarnoush,Sajad Jafari,Boshra Hatef,Matjaž Perc,Robert Repnik, Synchronizability of two neurons with switching in the coupling, Appl. Math. Comput.,350 (2019)

10. Bandyopadhyay A, Kar S, Impact of network structure on synchronization of Hindmarsh-Rose neurons coupled in structured network, Appl. Math. Comput., 333,194-212 (2018)

11. Fatemeh Parastesh,Chao-Yang Chen,Hamed Azarnoush,Sajad Jafari,Boshra Hatef, Synchronization patterns in a blinking multilayer neuronal network, Eur. Phys. J. Special Topics,228(11) (2019)

12. Budzinski R C,Boaretto B R R,Prado T L,Viana R L,Lopes $\mathrm{S} R$, Synchronous patterns and intermittency in a network induced by the rewiring of connections and coupling, Chaos,29(12) (2019)

13. Qingyun Wang,Zhisheng Duan,Matjaž Perc,Guanrong Chen, Synchronization transitions on small-world neuronal networks: Effects of information transmission delay and rewiring probability, Europhys. Lett.,5,83 (2008)

14. Toni Pérez, Guadalupe C., Garcia,Víctor M., Eguíluz, Raúl Vicente, Gordon Pipa, Claudio Mirasso, Effect of the Topology and Delayed Interactions in Neuronal Networks Synchronization, PLOS ONE,5,6 (2011)

15. Sun Xiaojuan,Perc Matjaž,Kurths Jürgen, Effects of partial time delays on phase synchronization in Watts-Strogatz small-world neuronal networks, Chaos,5,27 (2017)

16. Sun X, Li G, Synchronization transitions induced by partial time delay in a excitatory-inhibitory coupled neuronal network, Nonlinear Dynam., 89(4):2509-2520 (2017)

17. Bocheng Bao,Qinfeng Yang,Dong Zhu,Yunzhen Zhang, Quan $\mathrm{Xu}, \mathrm{Mo}$ Chen, Initial-induced coexisting and synchronous firing activities in memristor synapse-coupled Morris-Lecar bi-neuron network, Nonlinear Dynam., 99(3) (2020)

18. Ge Penghe,Cao Hongjun, Synchronization of Rulkov neuron networks coupled by excitatory and inhibitory chemical synapses, Chaos, 29(2) (2019)

19. Ge M, Jia Y, Xu Y, et al, Wave propagation and synchronization induced by chemical autapse in chain HindmarshRose neural network, Appl. Math. Comput., 352, 136-145 (2019)

20. J C Shi,M Luo,C S Huang, Dependence of synchronization transitions on mean field approach in two-way coupled neural system, Indian J. Phys., 92(8) (2018)

21. Ge M, Jia Y, Kirunda J B, et al, Propagation of firing rate by synchronization in a feed-forward multilayer HindmarshRose neural network, Neurocomputing, 320(3).60-68 (2018)

22. Gopal R,Chandrasekar V K, Venkatesan A,Lakshmanan M, Observation and characterization of chimera states in coupled dynamical systems with nonlocal coupling, Phys. rev. E, 89(5) (2014)

23. Kim Sang-Yoon,Lim Woochang, Burst synchronization in a scale-free neuronal network with inhibitory spike-timingdependent plasticity, Cogn. neurodynamics, 13(1) (2019)

24. Dong J, Zhang GJ, Xie Y, Yao H, and Wang J, Dynamic behavior analysis of fractional-order Hindmarsh-Rose neuronal model, Cogn. Neurodynamics 8, 167-175, (2014)

25. Giresse T A, Crepin K T, Martin T, Generalized synchronization of the extended Hindmarsh-Rose neuronal model with fractional order derivative, Chaos Soliton. Fract., 118, 311-319 (2018)

26. Fanqi Meng,Xiaoqin Zeng,Zuolei Wang,Xinjun Wang, Adaptive Synchronization of Fractional-Order Coupled Neurons Under Electromagnetic Radiation, Int. J. Bifurcat. Chaos, 30(03) (2019)

27. Fanqi Meng,Xiaoqin Zeng,Zuolei Wang, Dynamical behavior and synchronization in time-delay fractional-order coupled neurons under electromagnetic radiation, Nonlinear Dynam., 95(2) (2019)

28. Li R, Chaotic synchronization of a class of fractional-order system, 77. Air Force Engineering University, XiAn (2014)

29. Mi Lv,Jun Ma, Multiple modes of electrical activities in a new neuron model under electromagnetic radiation, Neurocomputing, 205 (2016)

30. Lv M, Wang C, Ren G, et al, Model of electrical activity in a neuron under magnetic flow effect, Nonlinear Dynam., 85(3), 1479-1490 (2016)

31. A.S. Etémé, C.B. Tabi, A. Mohamadou, Firing and synchronization modes in neural network under magnetic stimulation, Commun. Nonlinear Sci., 72 (2019)

32. Tingting Fang, Jiqian Zhang, Shoufang Huang, Fei $\mathrm{Xu}$,Maosheng Wang, Hang Yang, Synchronous behavior among different regions of the neural system induced by electromagnetic radiation, Nonlinear Dynam., 98(2) (2019)

33. Jun Ma, Fuqiang Wu, Chunni Wang, Synchronization behaviors of coupled neurons under electromagnetic radiation, International Journal of Modern Physics B, 31(2) (2017)

34. Armand S. Etémé,Conrad B. Tabi,Alidou Mohamadou,Timoléon C. Kofané, Long-range memory effects in a magnetized Hindmarsh-Rose neural network, Commun. Nonlinear Sci., 84 (2020)

35. Diethelm K, Ford N J, Freed A D. A Predictor-Corrector Approach for the Numerical Solution of Fractional Differential Equations, Nonlinear Dynam., 29(1-4), 3-22 (2002)

36. G. Adomian, A Review of The Decomposition Method and Some Recent Results for Nonlinear Equations, Computers Math. Applic., 21(5), 101-127 (1991)

37. Shaobo He, Kehui Sun, Huihai Wang, Solution of the fractional-order chaotic system based on Adomian decomposition algorithm and its complexity analysis, Acta phys. Sin., 63(3) (2014) 
Figures

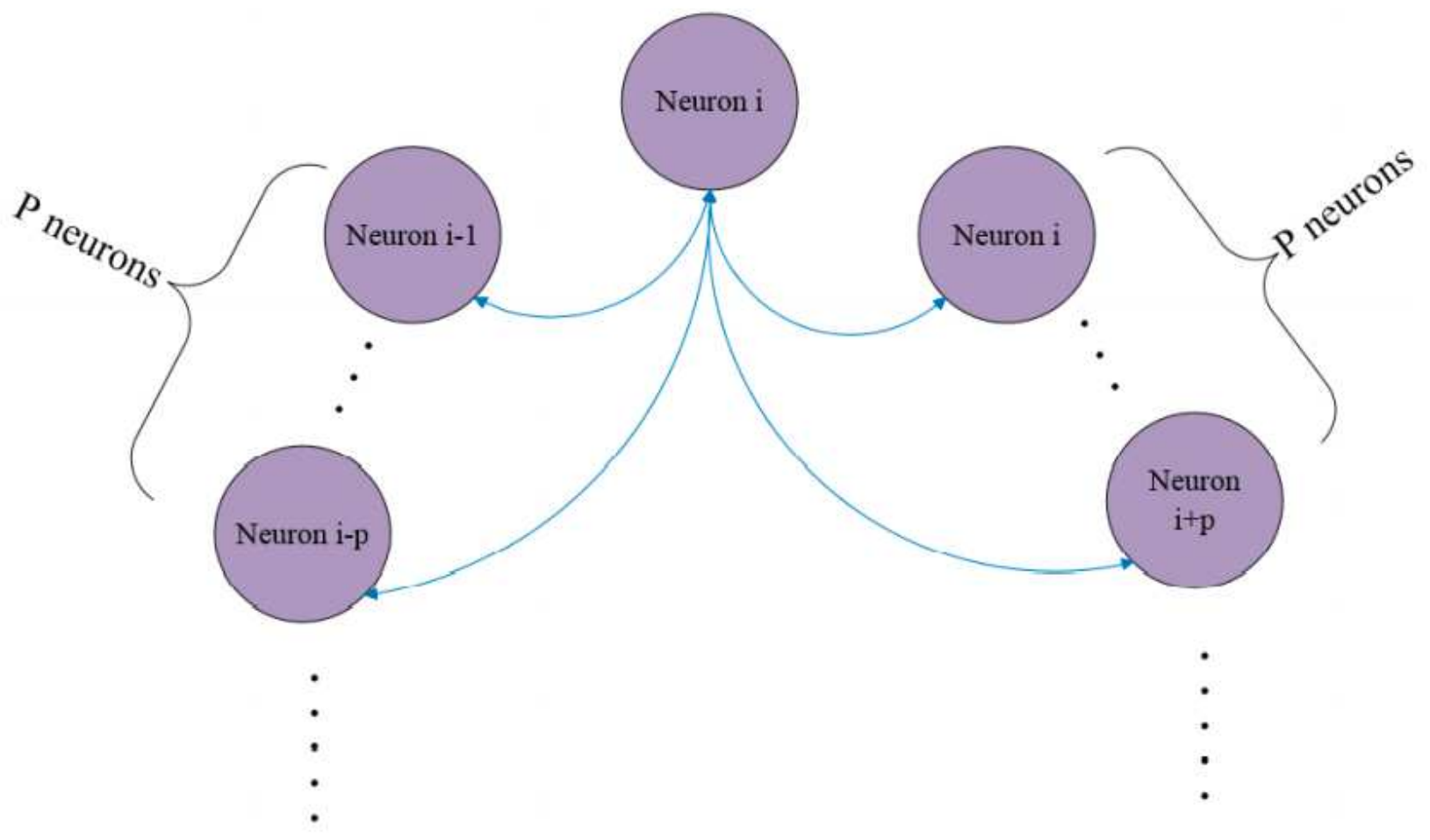

Figure 1

Please see the Manuscript PDF file for the complete figure caption 


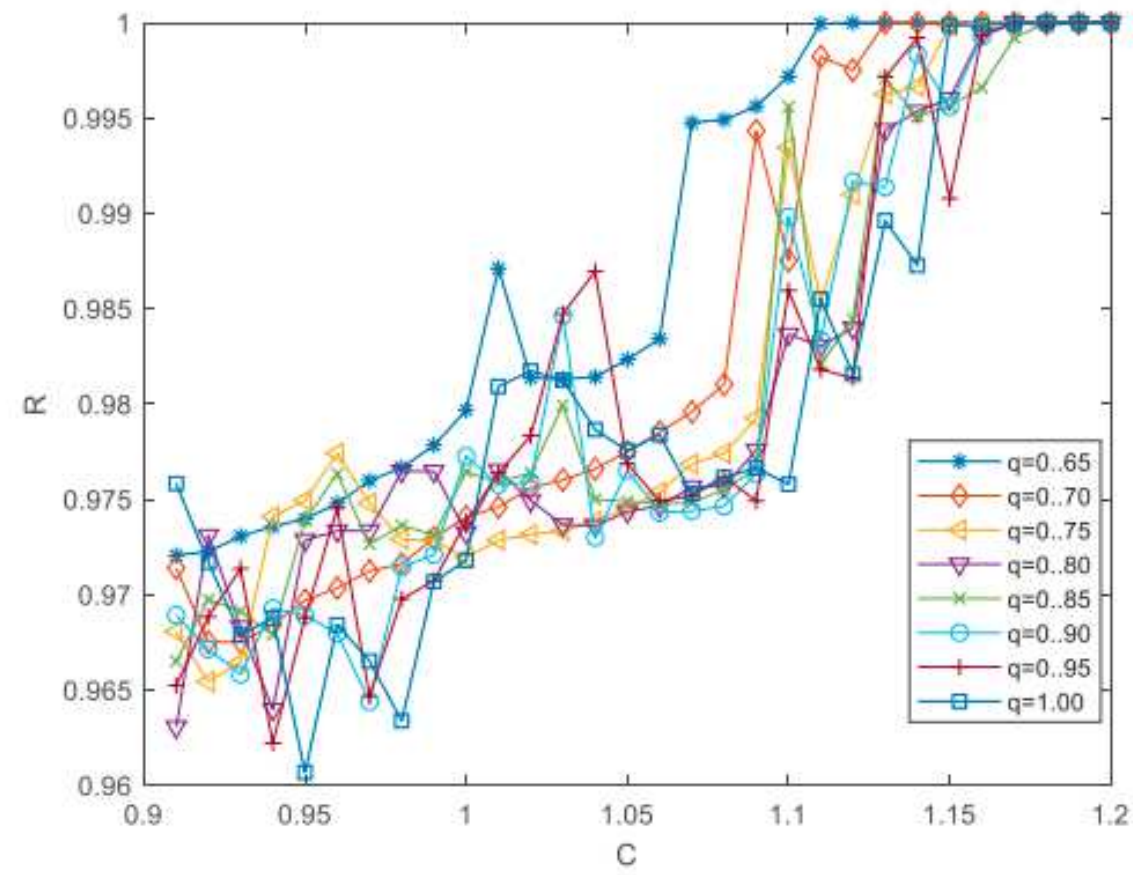

(a)

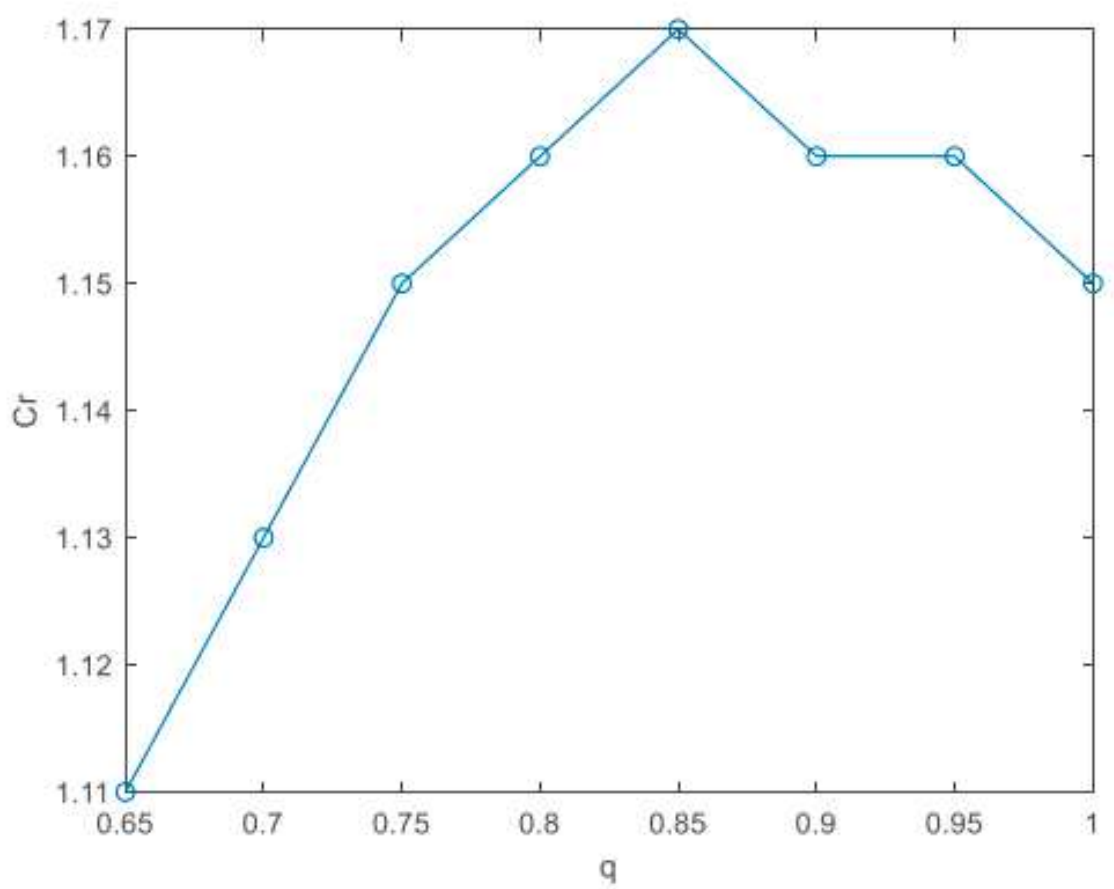

(b)

\section{Figure 2}

Please see the Manuscript PDF file for the complete figure caption 


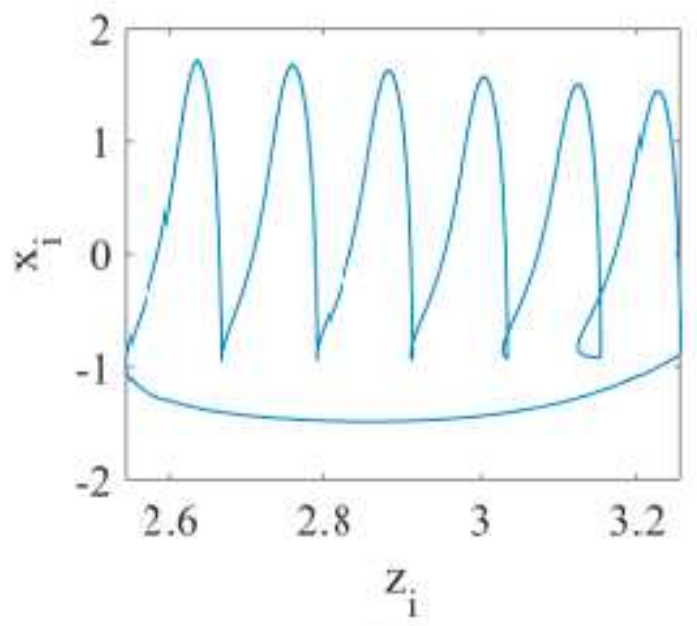

(a)

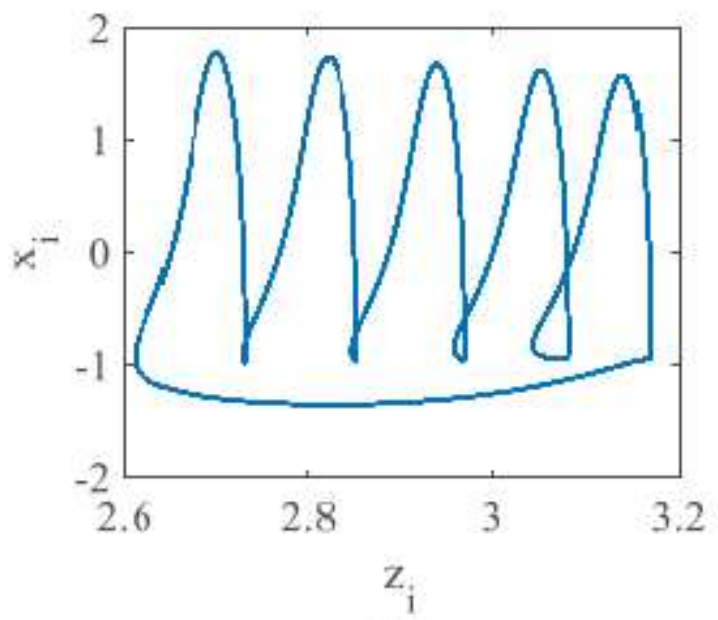

(c)

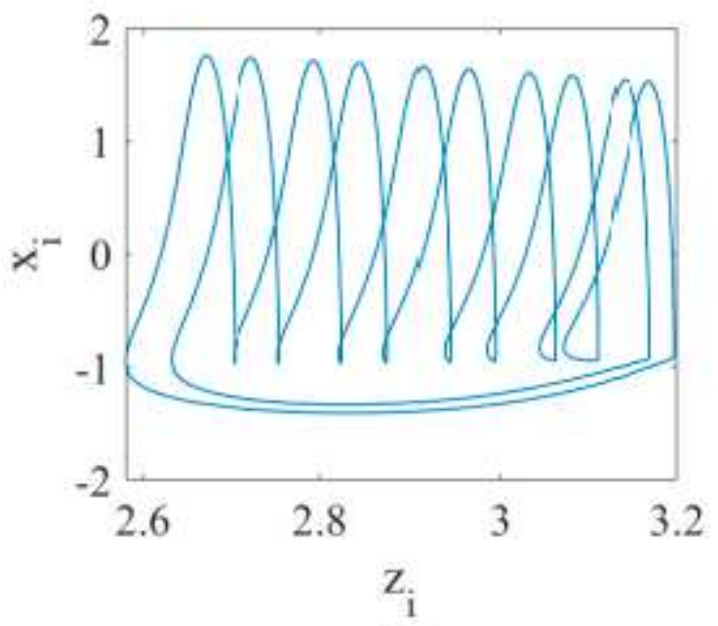

(b)

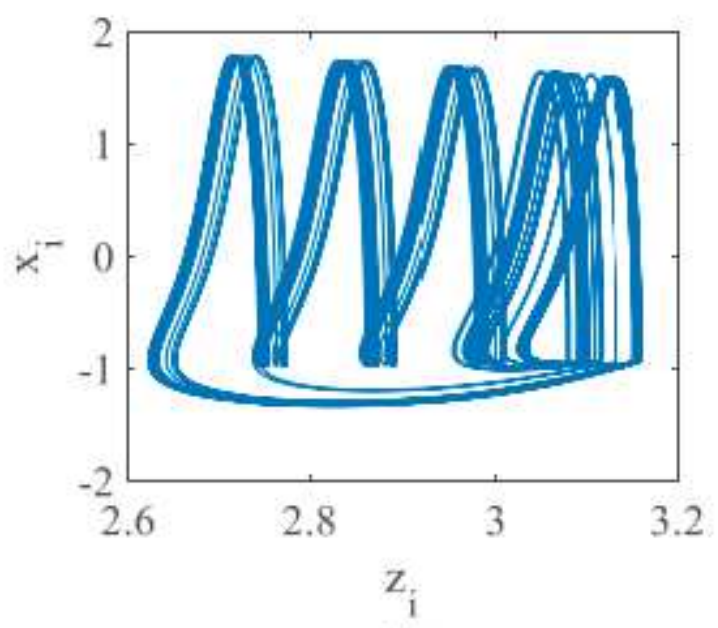

(d)

Figure 3

Please see the Manuscript PDF file for the complete figure caption 


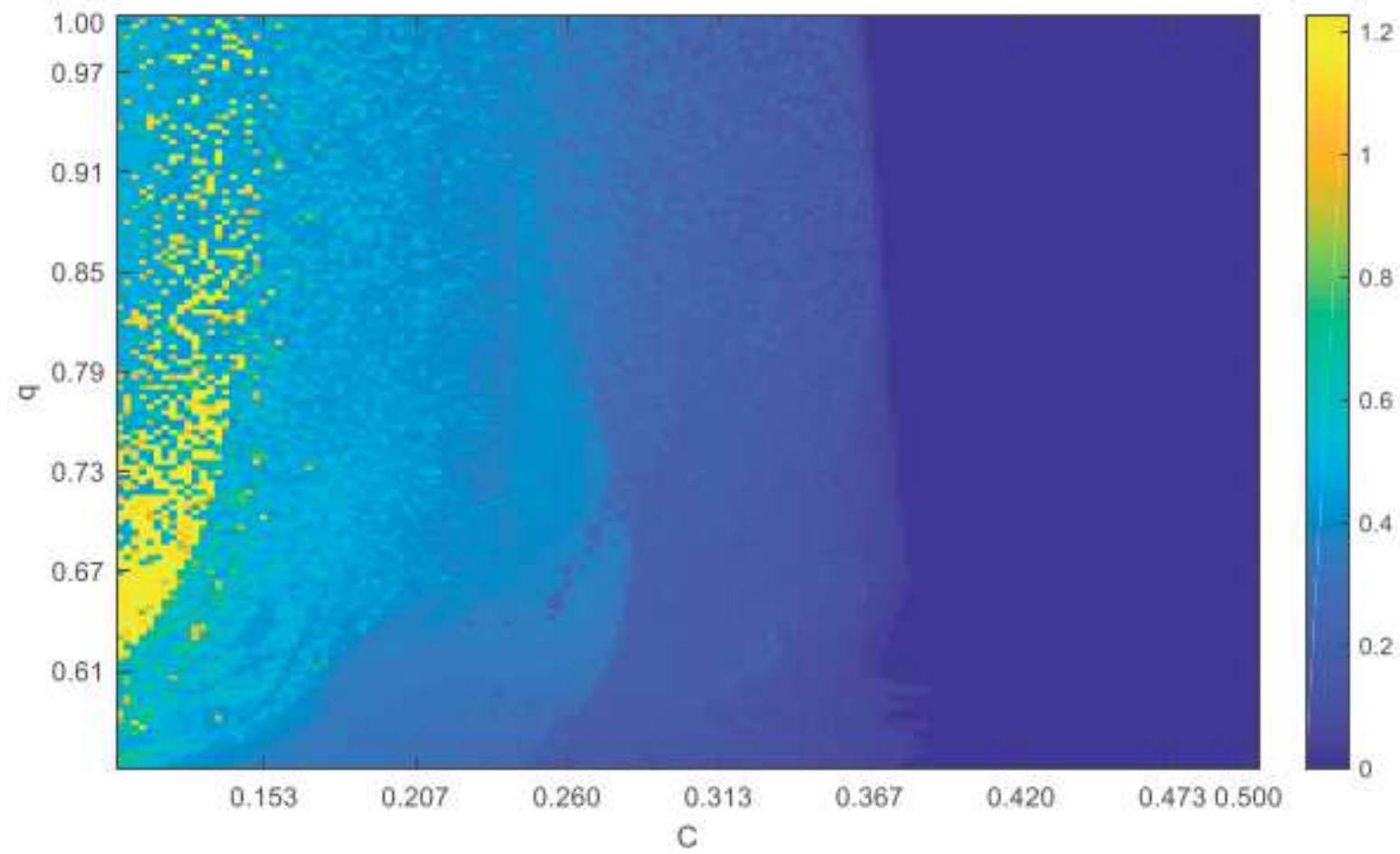

Figure 4

Please see the Manuscript PDF file for the complete figure caption 


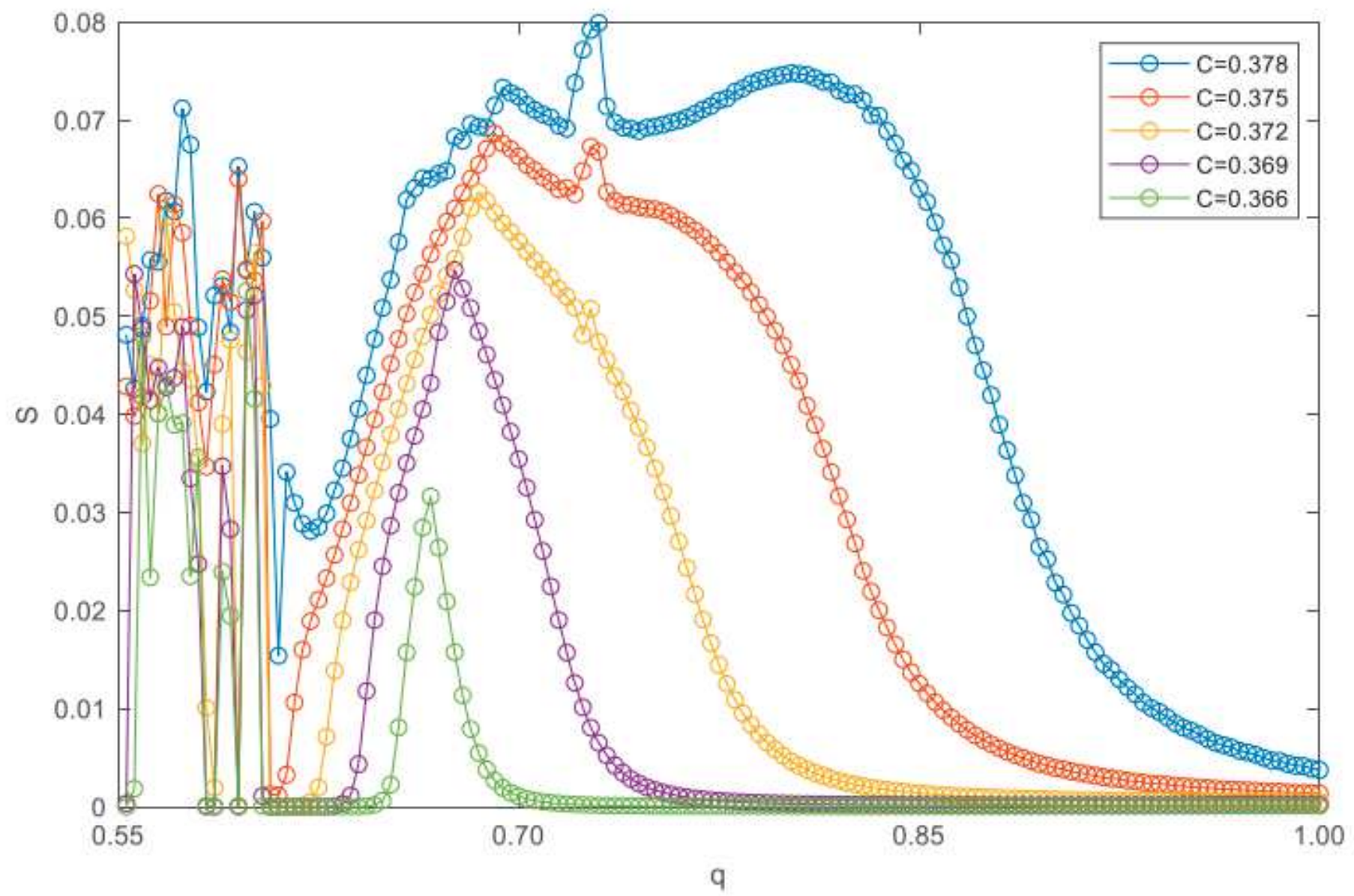

Figure 5

Please see the Manuscript PDF file for the complete figure caption 


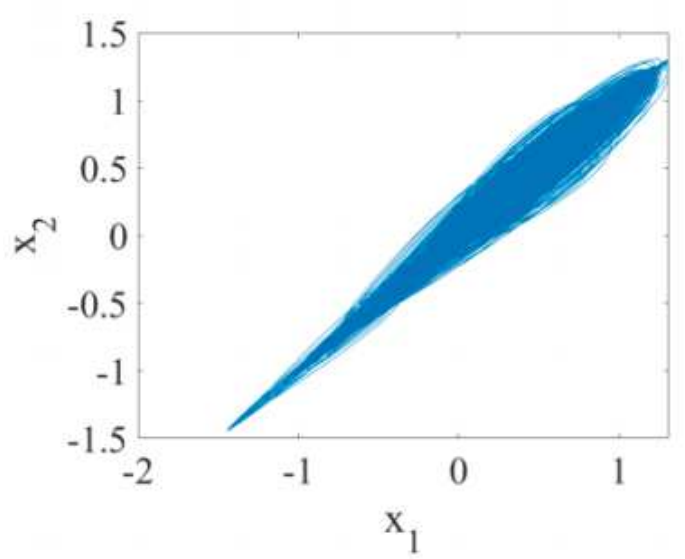

(a)

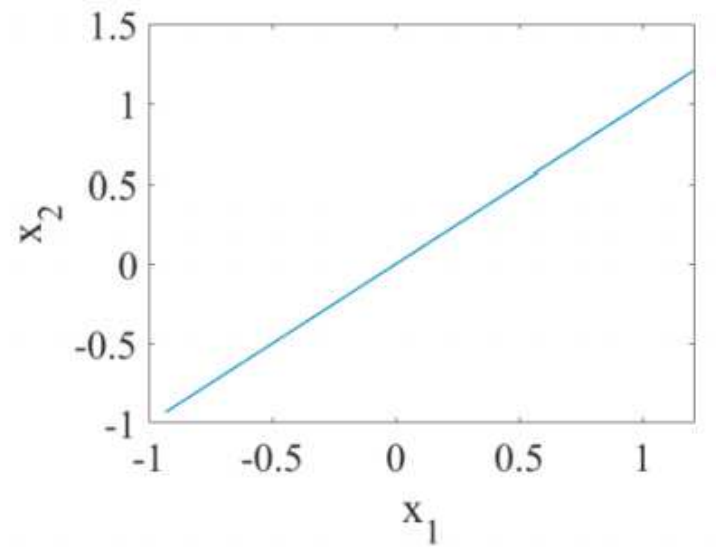

(c)

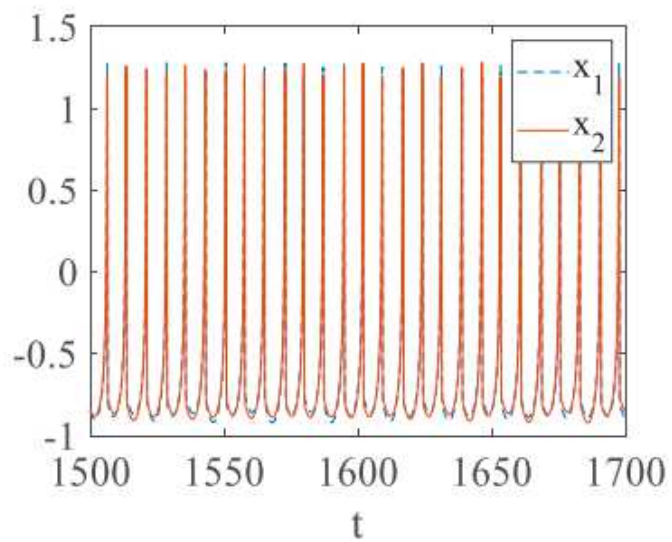

(e)

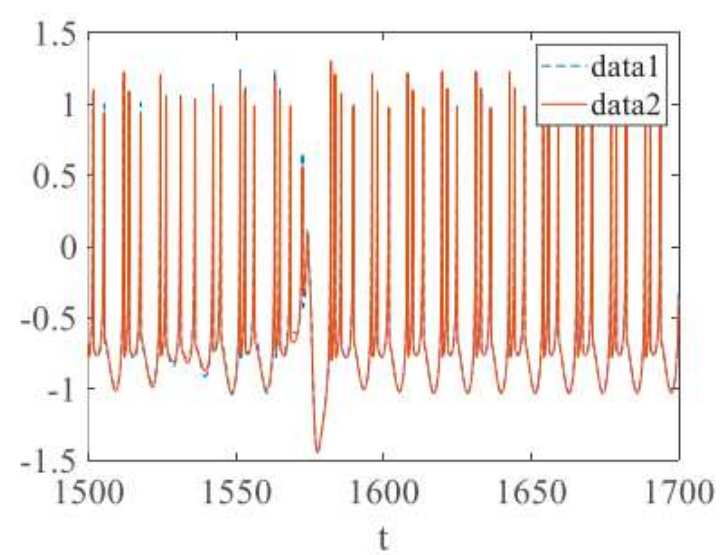

(b)

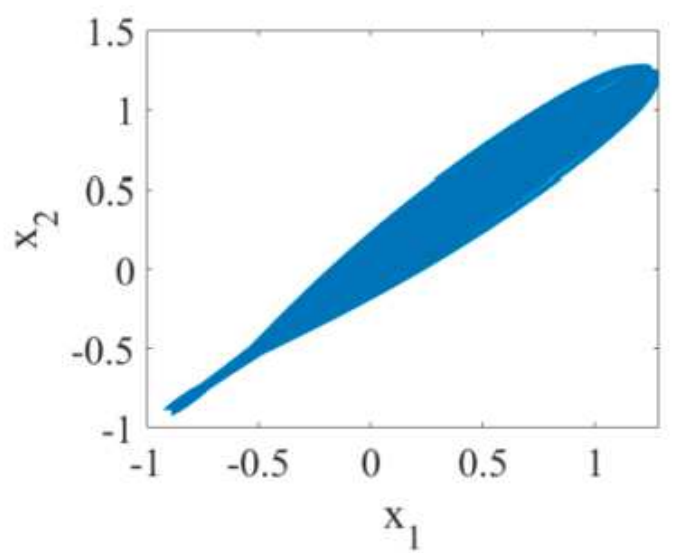

(d)

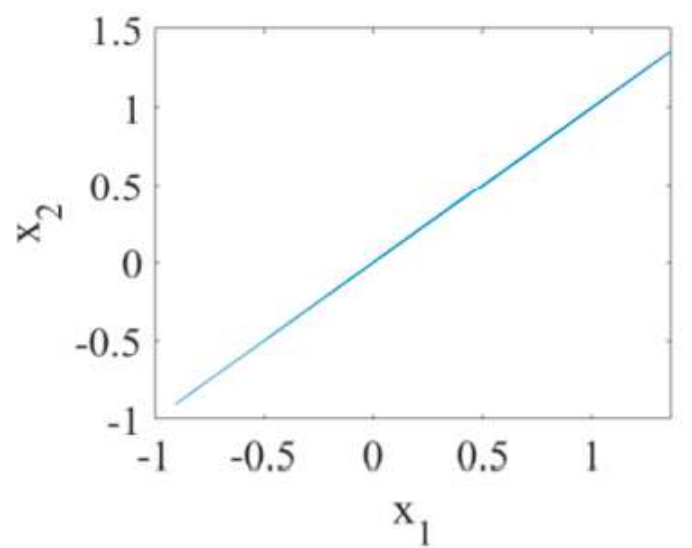

(f)

\section{Figure 6}

Please see the Manuscript PDF file for the complete figure caption 


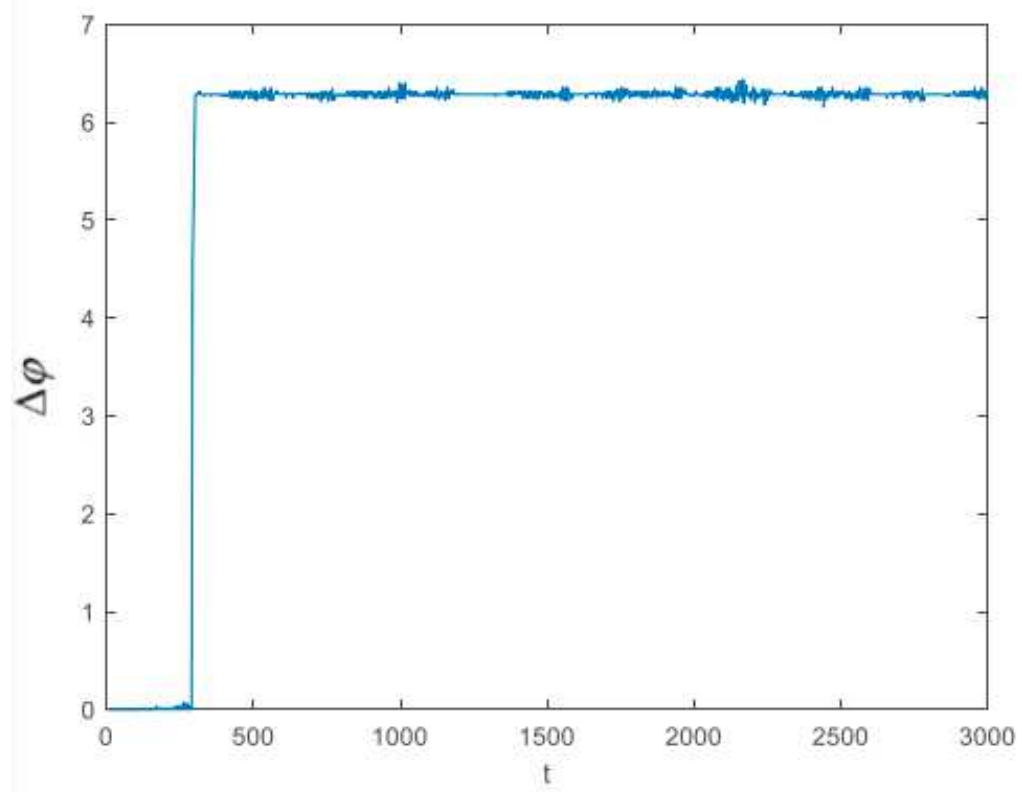

(a)

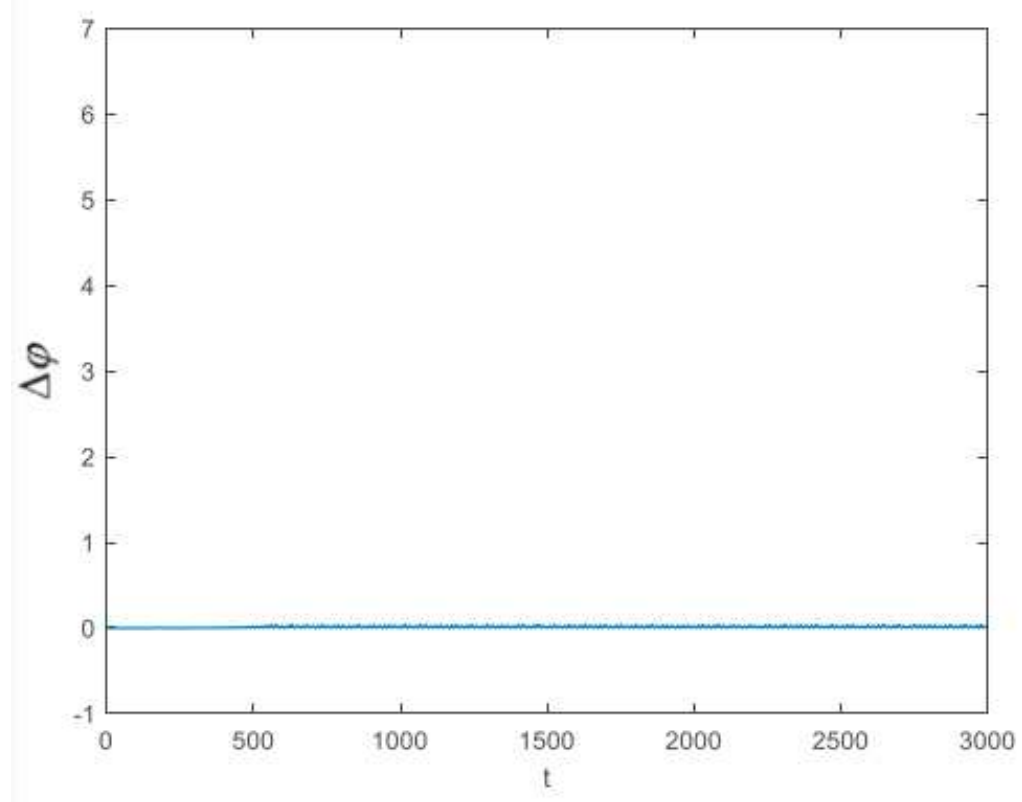

(b)

\section{Figure 7}

Please see the Manuscript PDF file for the complete figure caption 


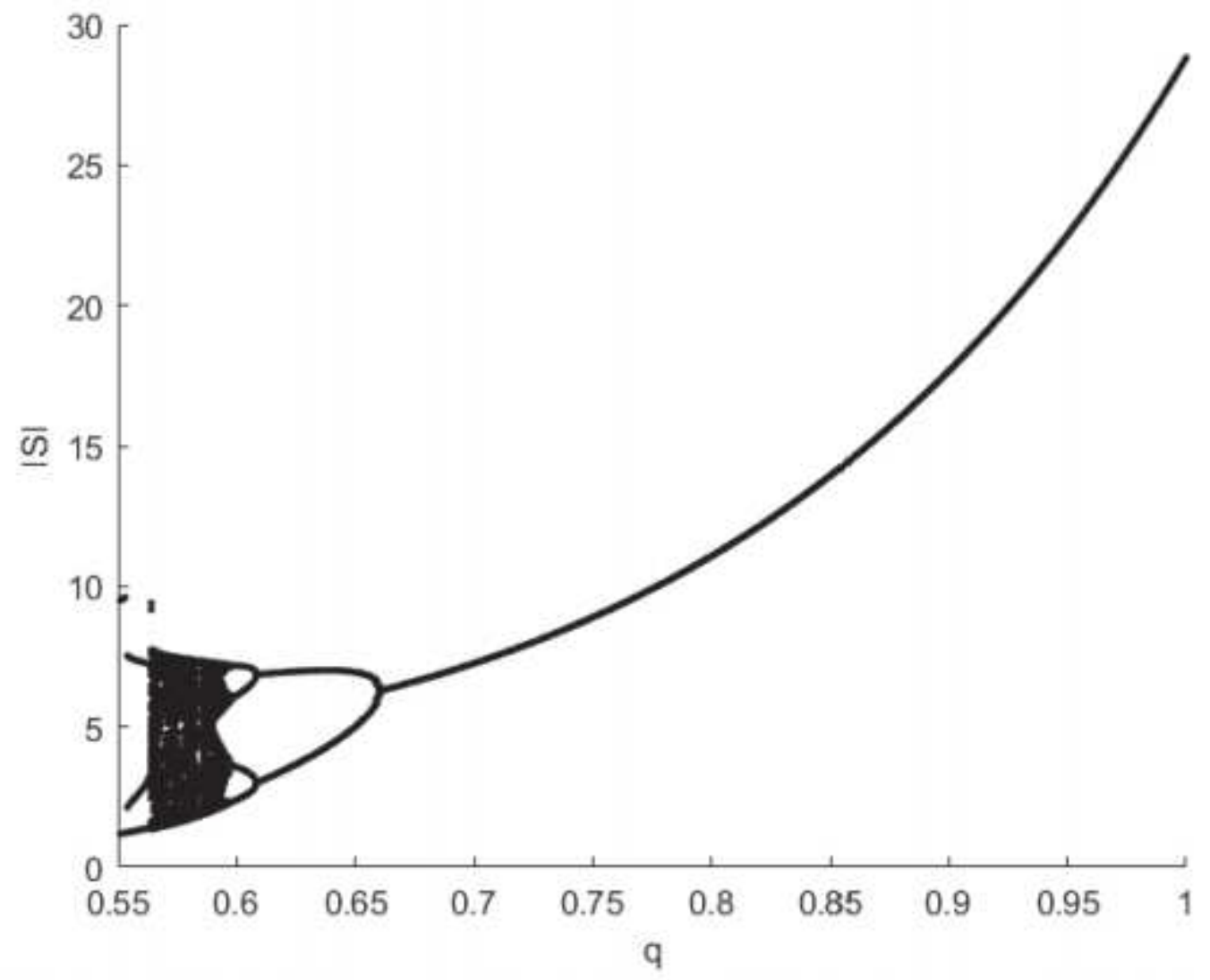

Figure 8

Please see the Manuscript PDF file for the complete figure caption 


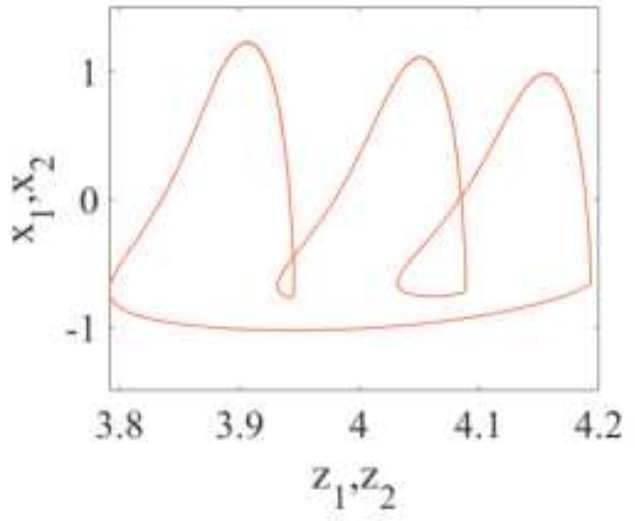

(a)

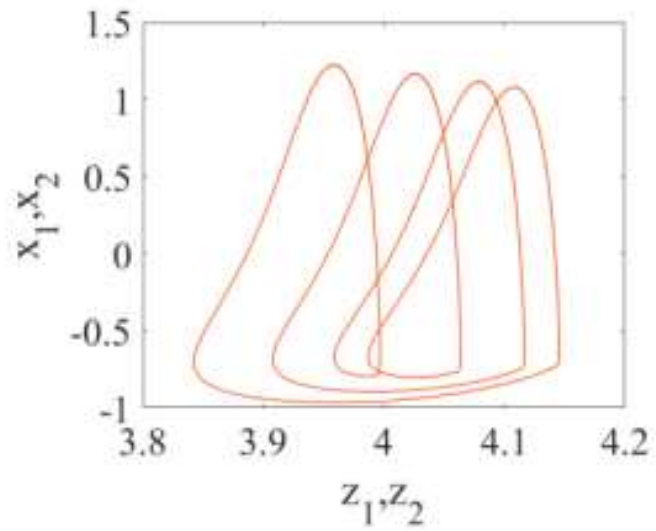

(c)

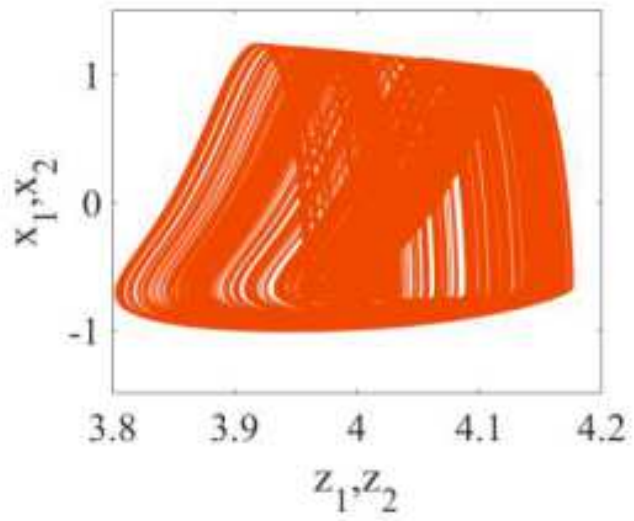

(b)

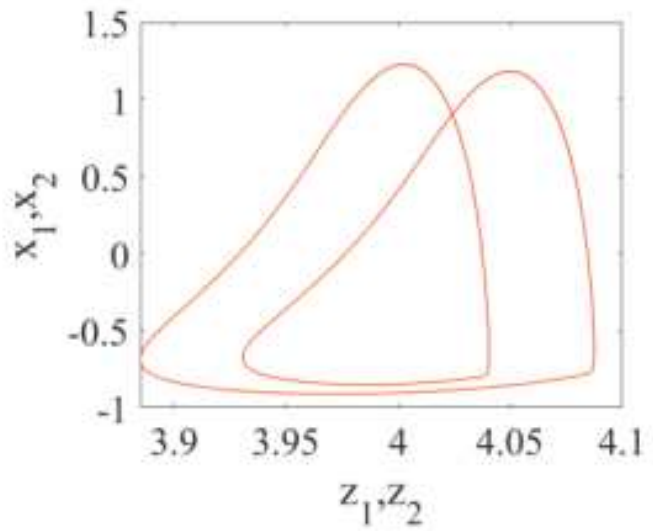

(d)

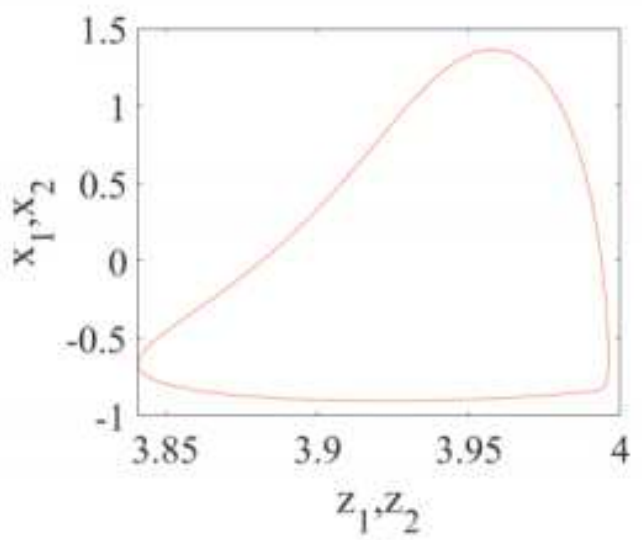

(e)

Figure 9

Please see the Manuscript PDF file for the complete figure caption 


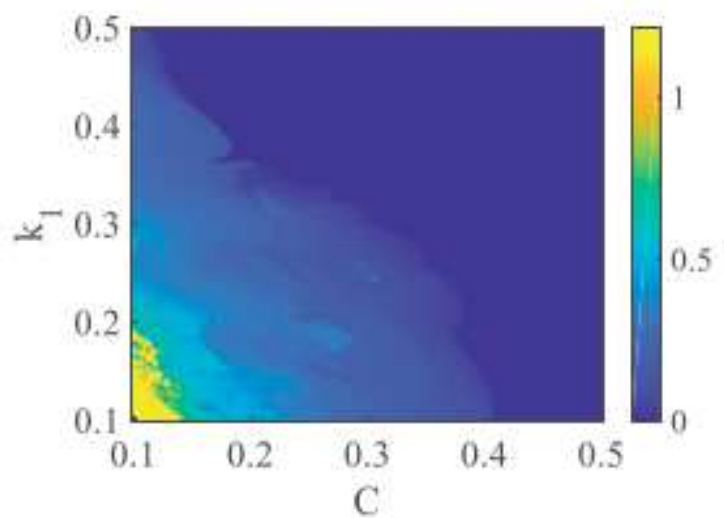

(a)

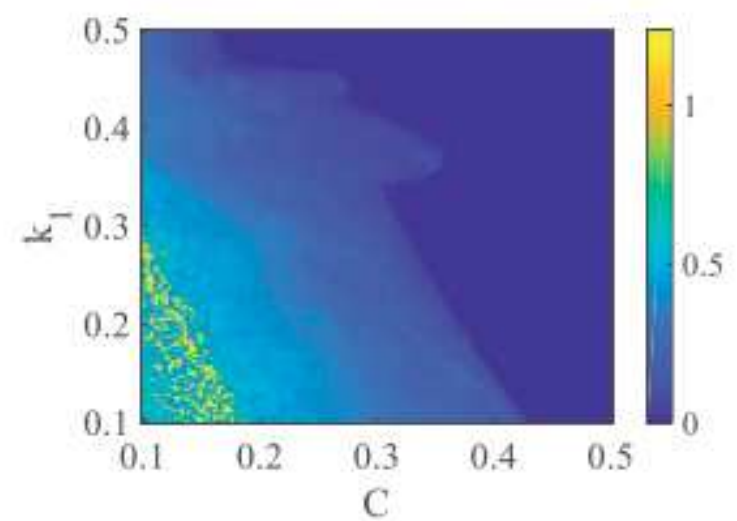

(b)

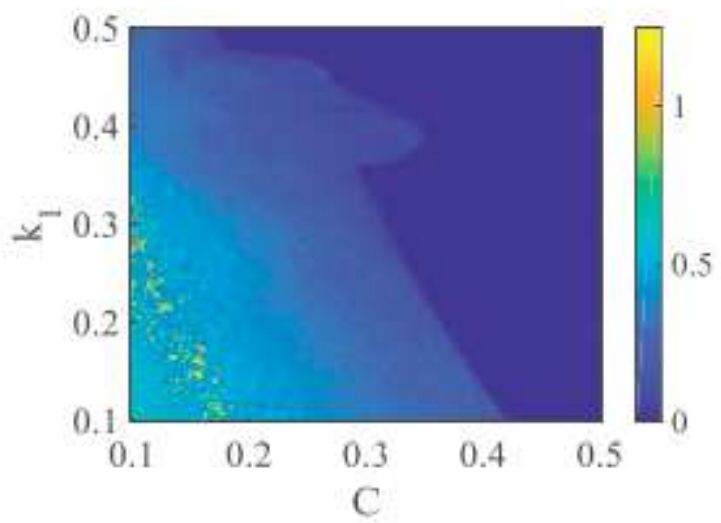

(c)

Figure 10

Please see the Manuscript PDF file for the complete figure caption 


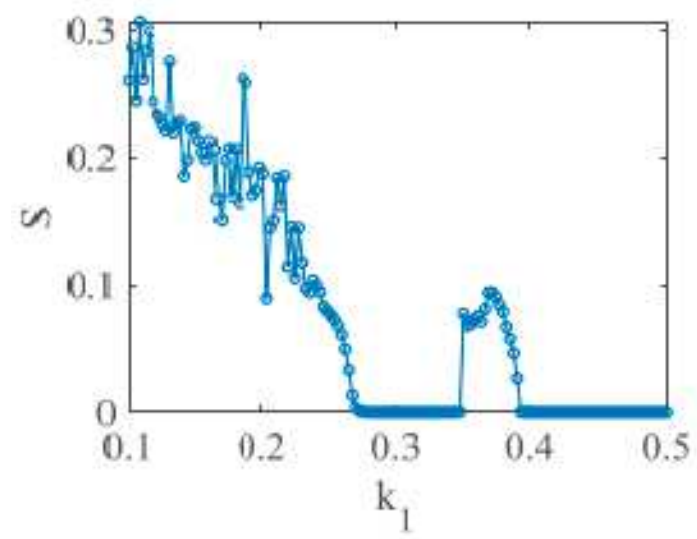

(a)

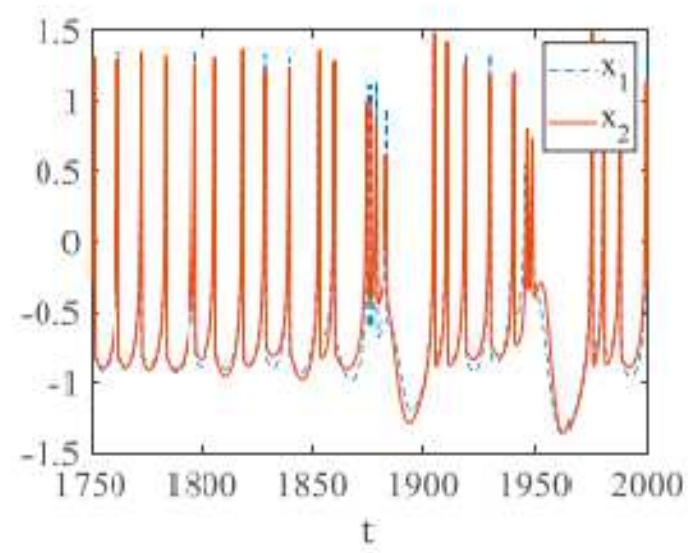

(c)

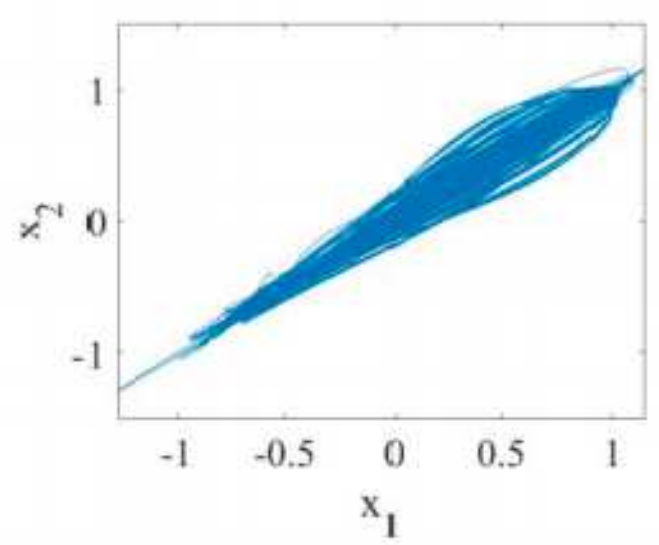

(e)

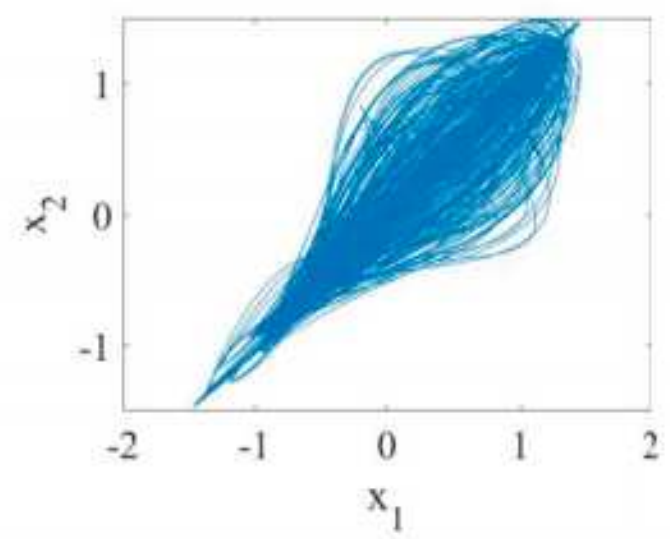

(b)

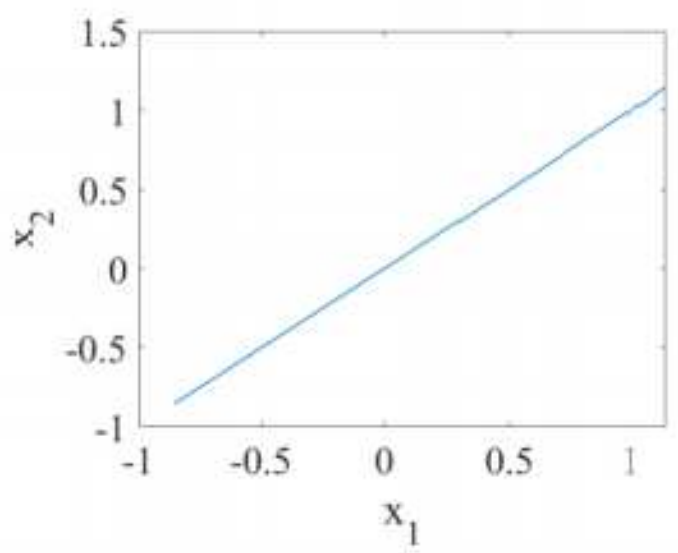

(d)

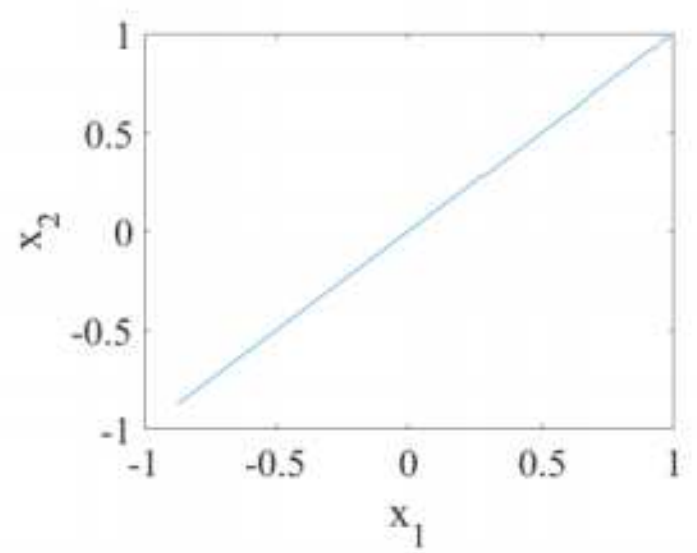

(f)

\section{Figure 11}

Please see the Manuscript PDF file for the complete figure caption 


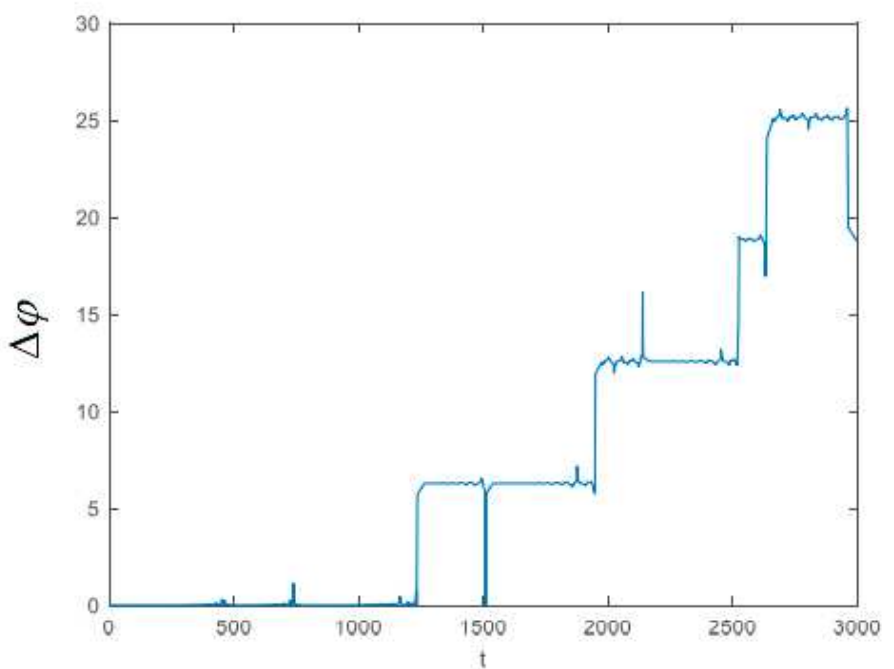

(a)

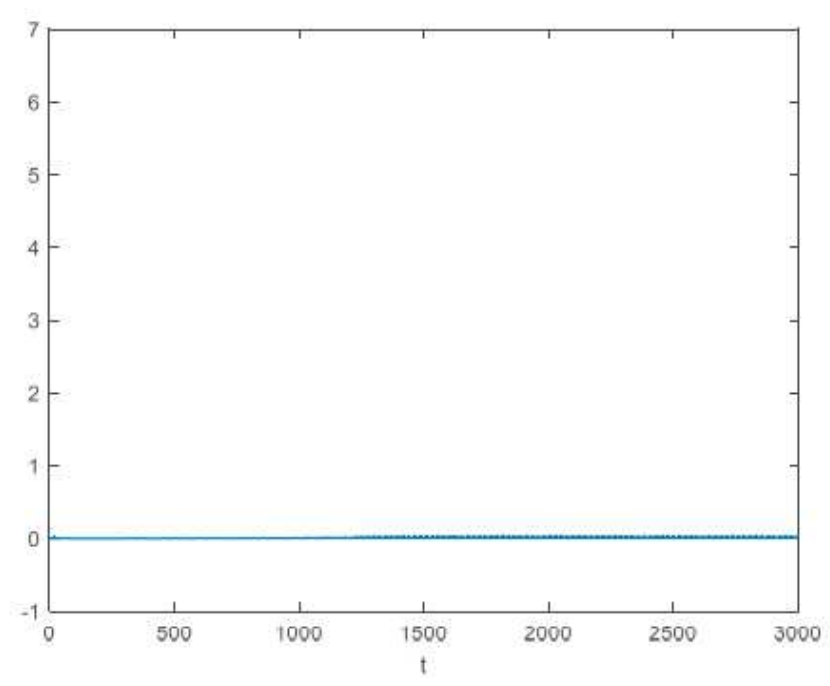

(b)

\section{Figure 12}

Please see the Manuscript PDF file for the complete figure caption

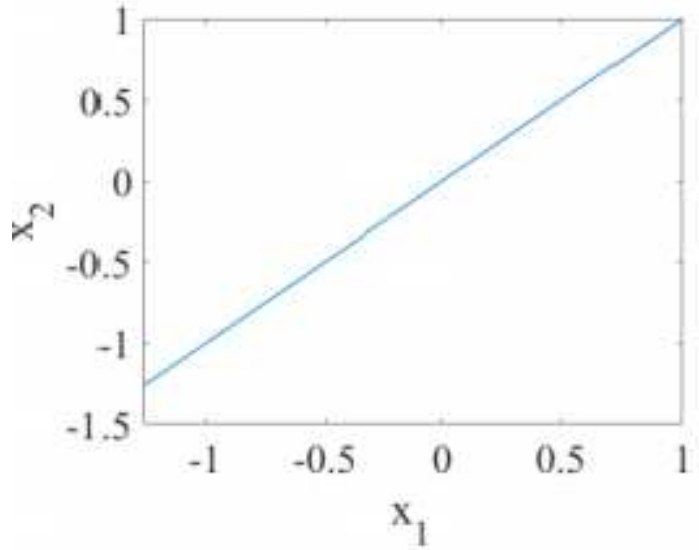

(a)

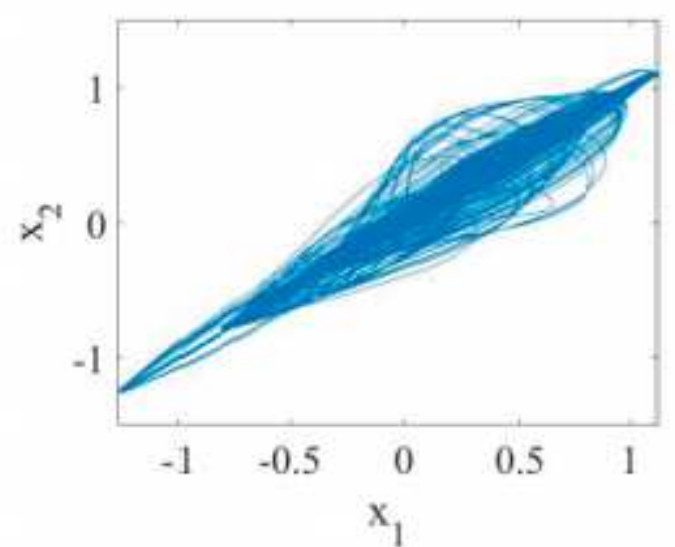

(b)

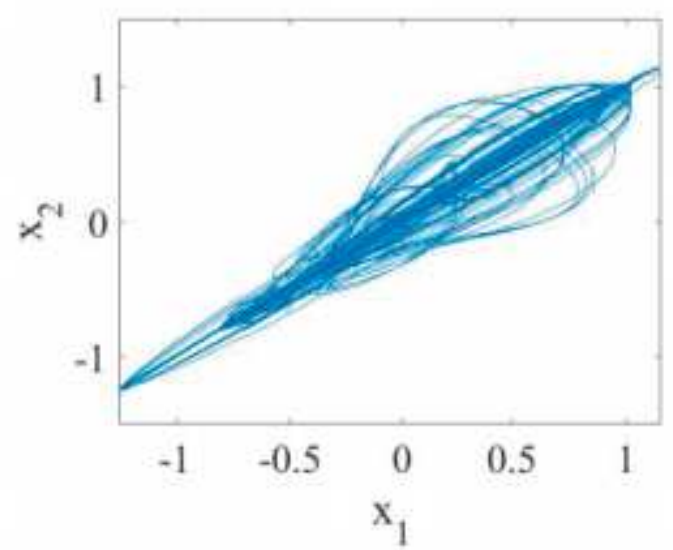

(c) 
Figure 13

Please see the Manuscript PDF file for the complete figure caption

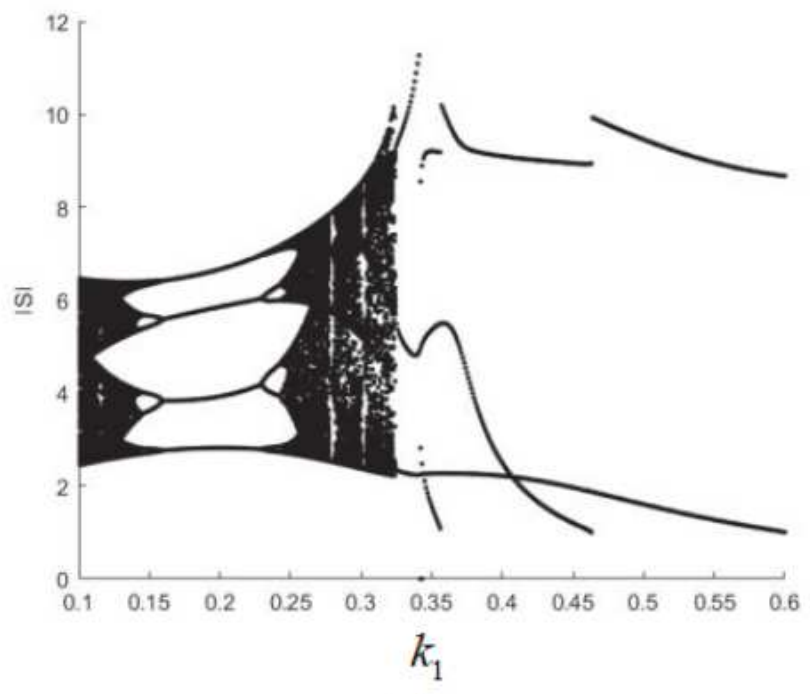

(a)

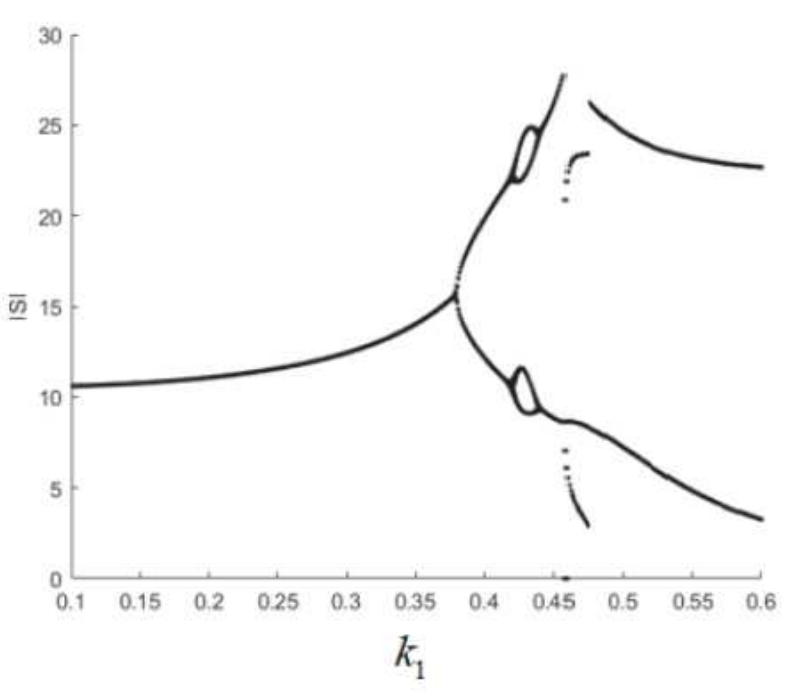

(b)

\section{Figure 14}

Please see the Manuscript PDF file for the complete figure caption 


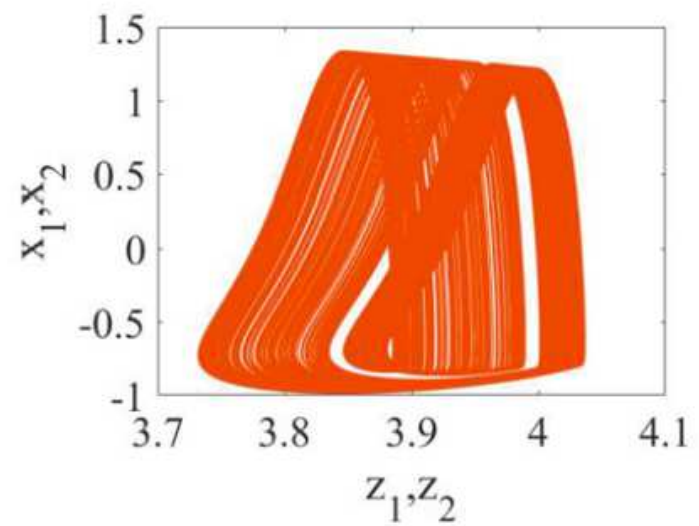

(a)

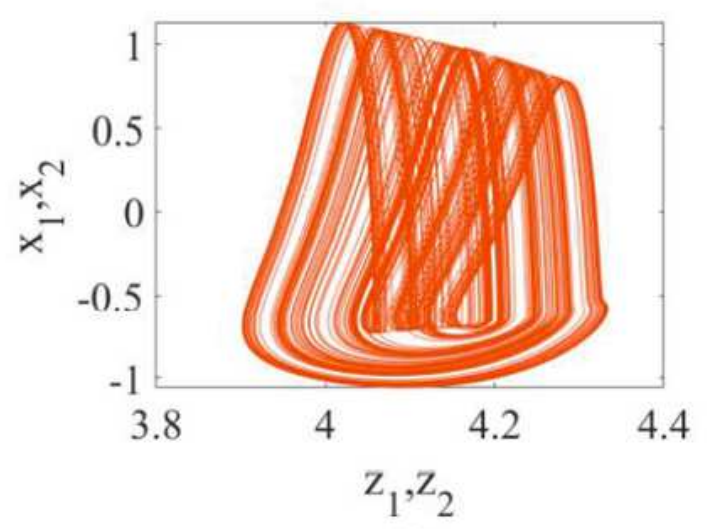

(c)

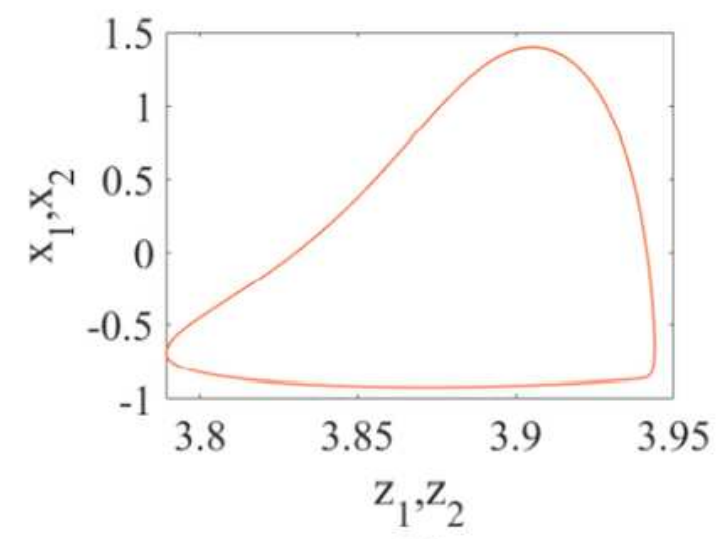

(e)

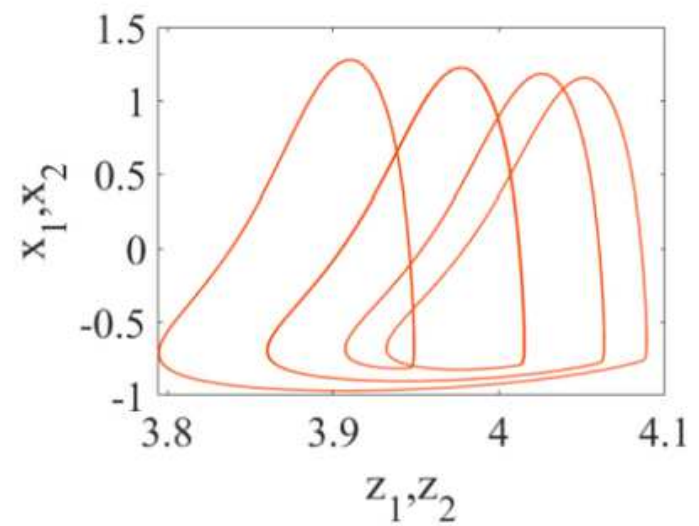

(b)

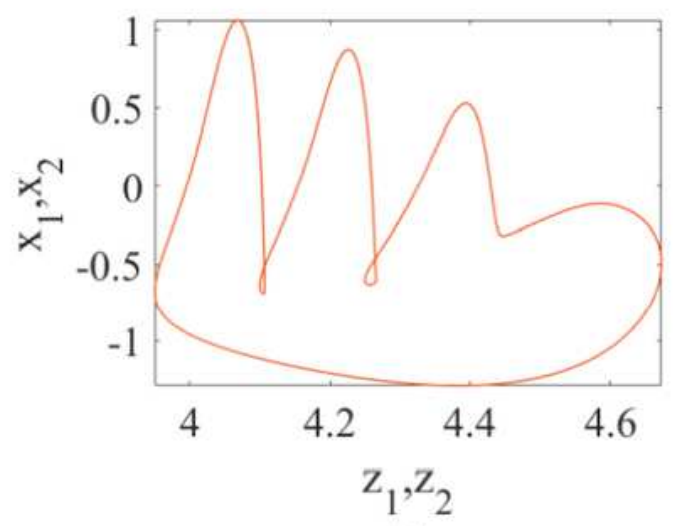

(d)

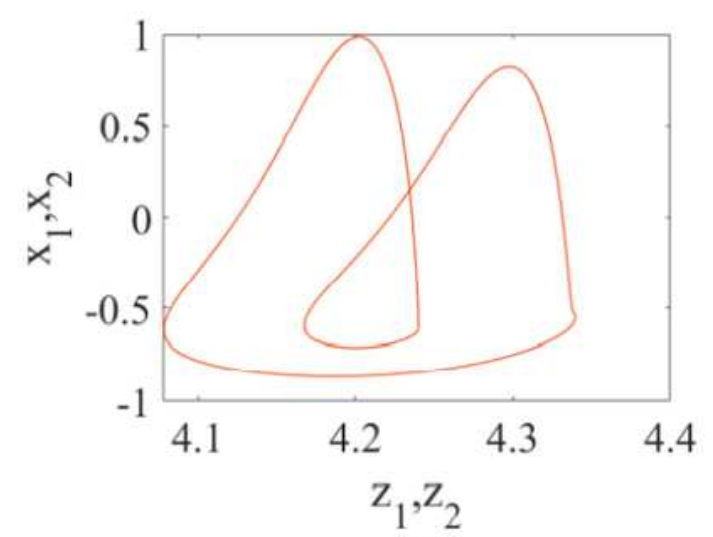

(f)

\section{Figure 15}

Please see the Manuscript PDF file for the complete figure caption 


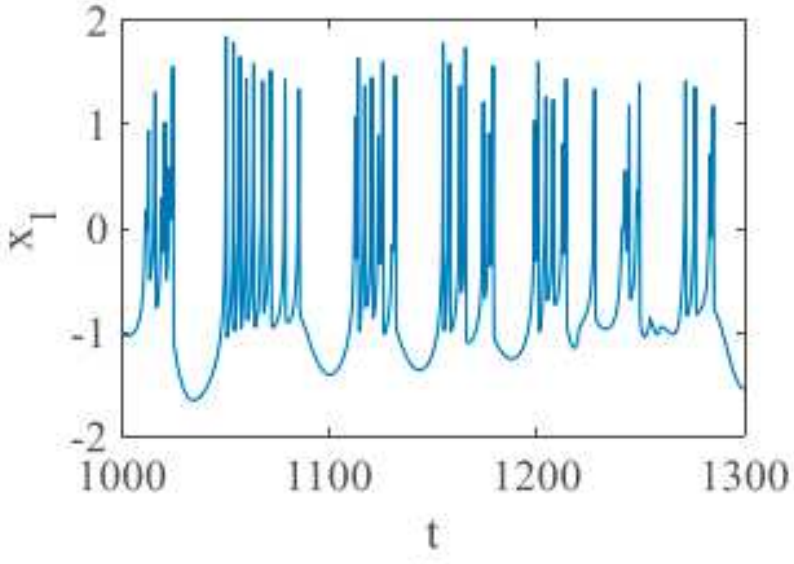

(a)

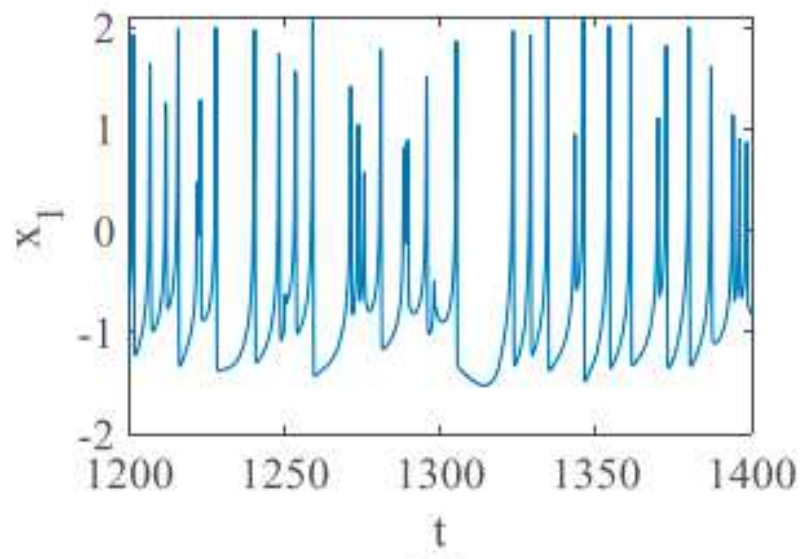

(b)

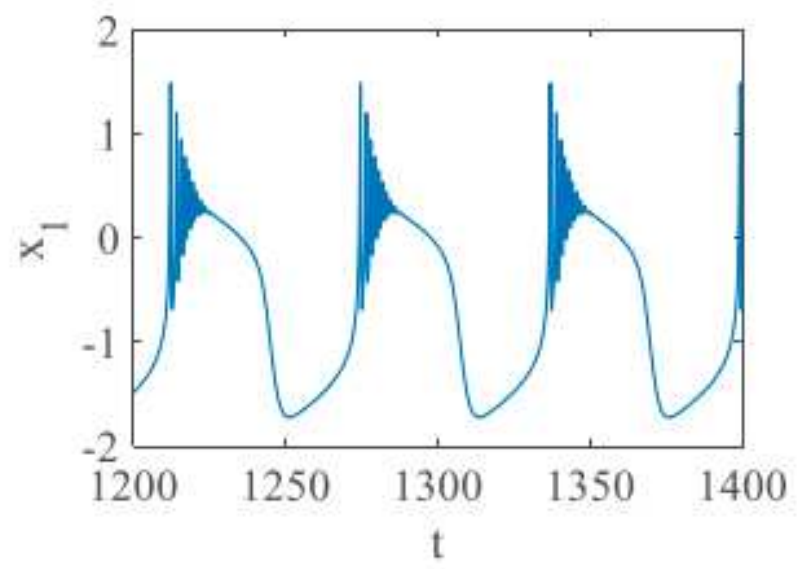

(c)

Figure 16

Please see the Manuscript PDF file for the complete figure caption 


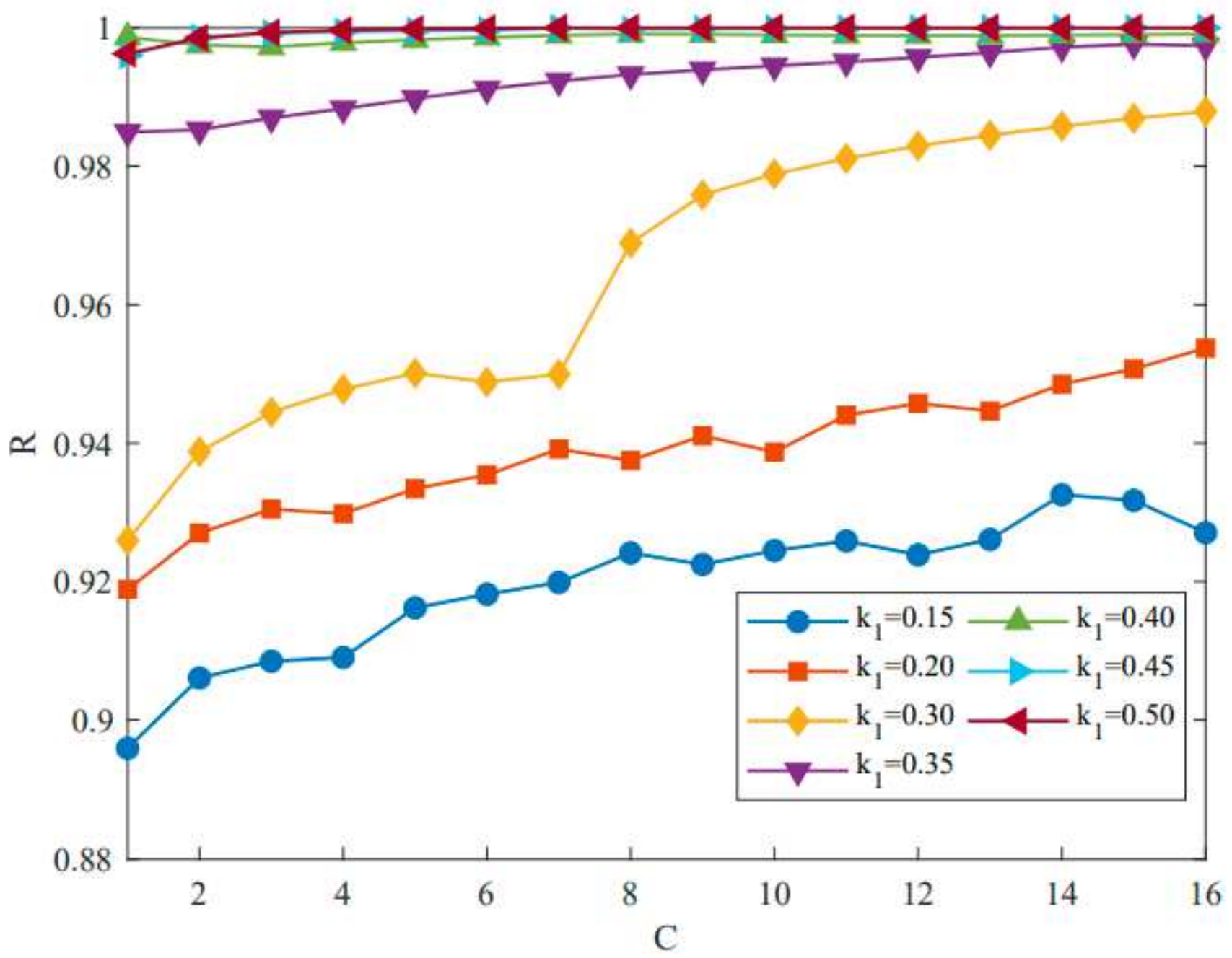

Figure 17

Please see the Manuscript PDF file for the complete figure caption 


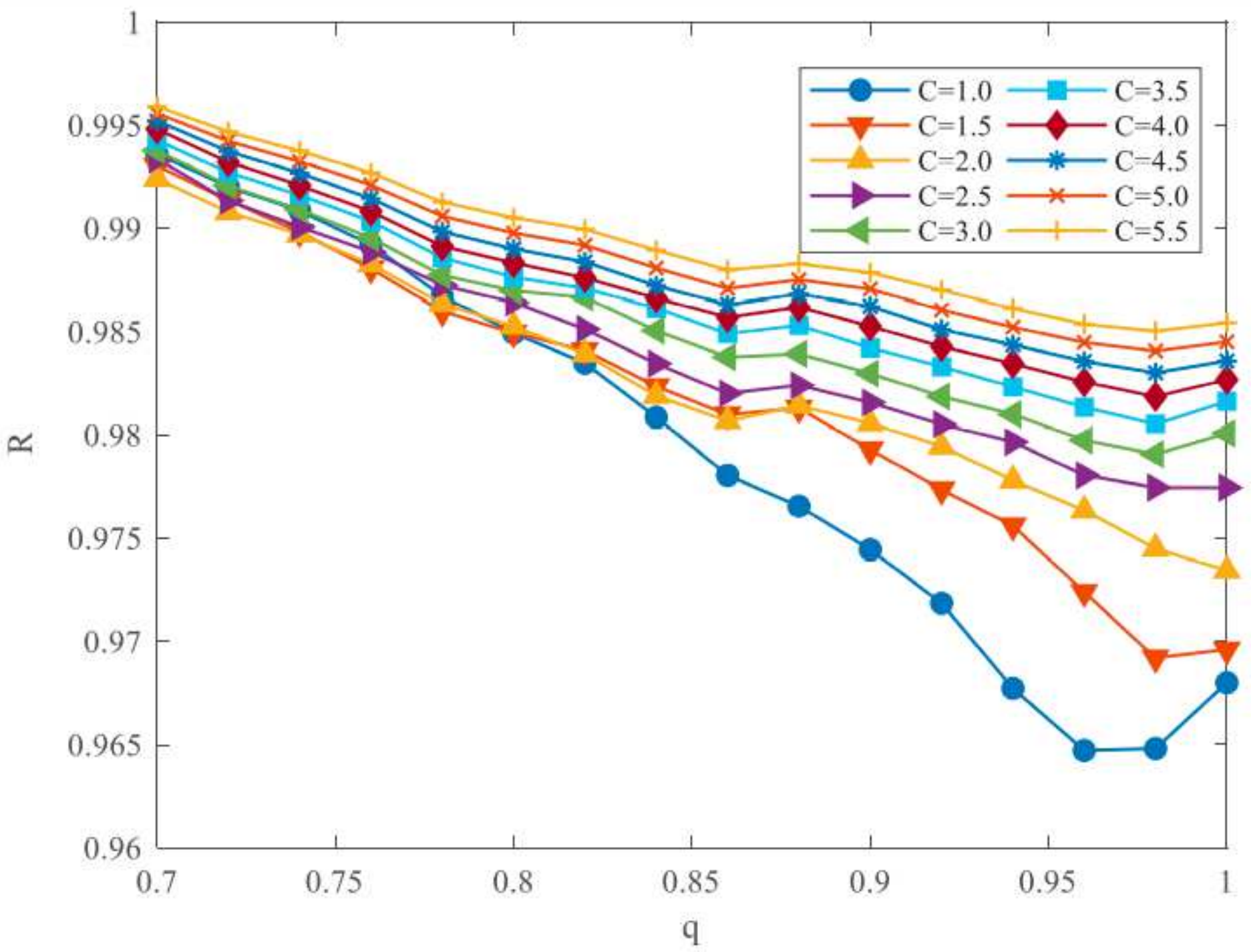

Figure 18

Please see the Manuscript PDF file for the complete figure caption 


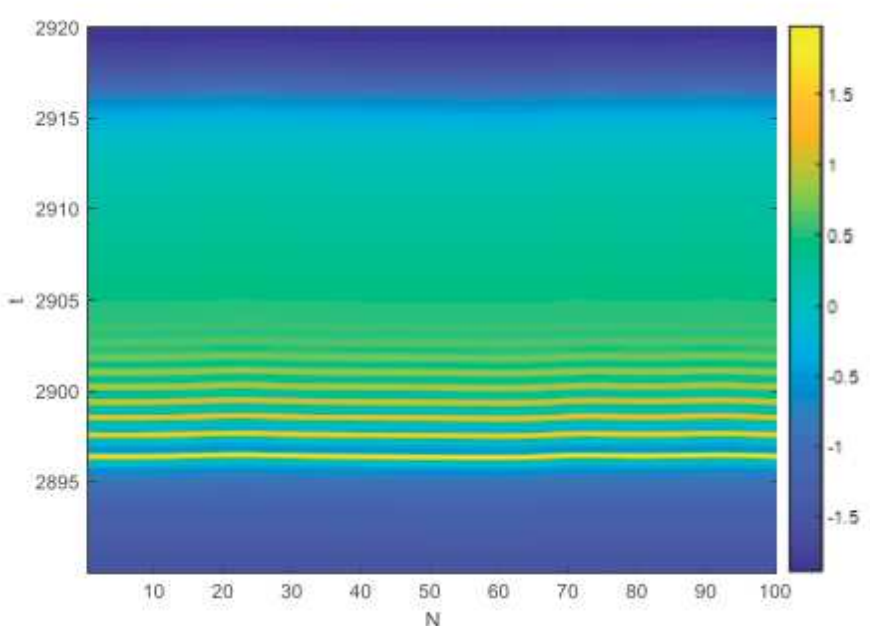

(a)

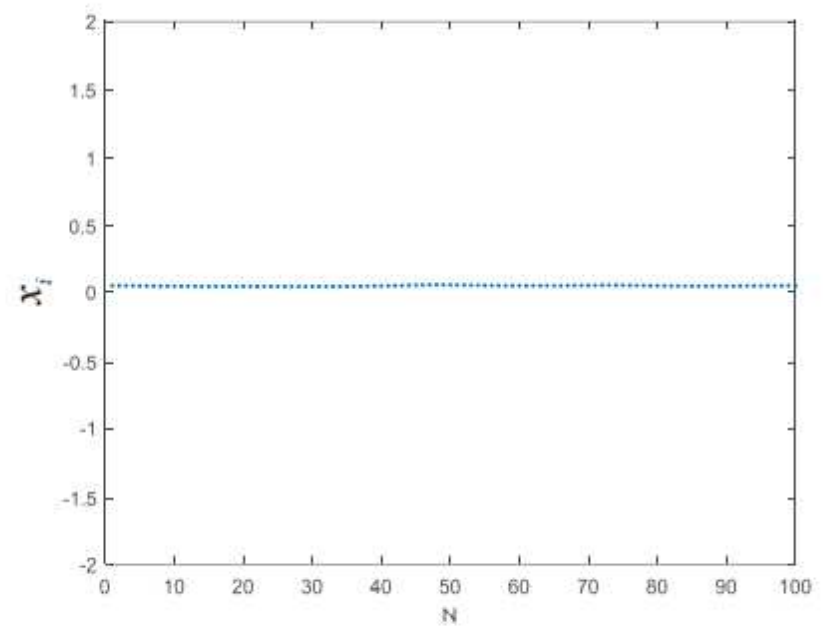

(c)

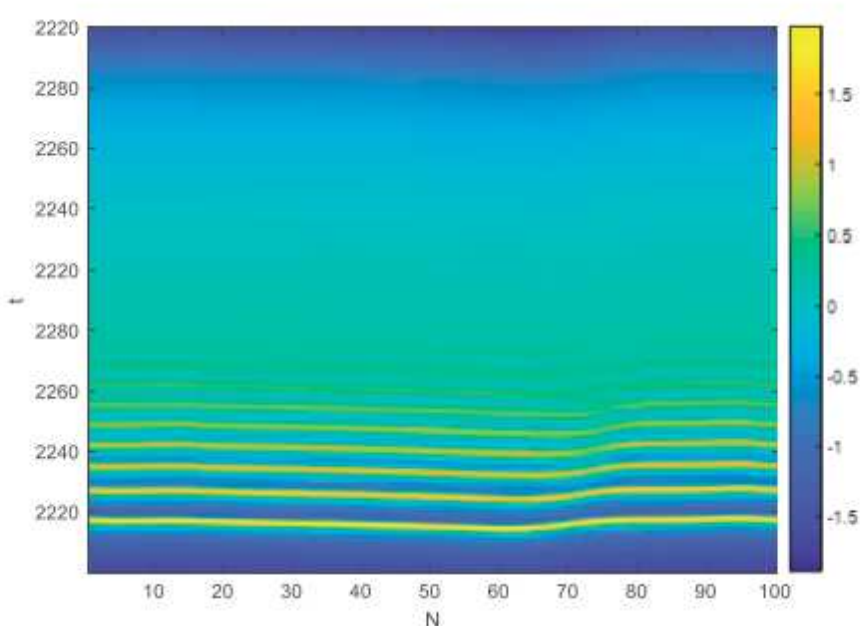

(b)

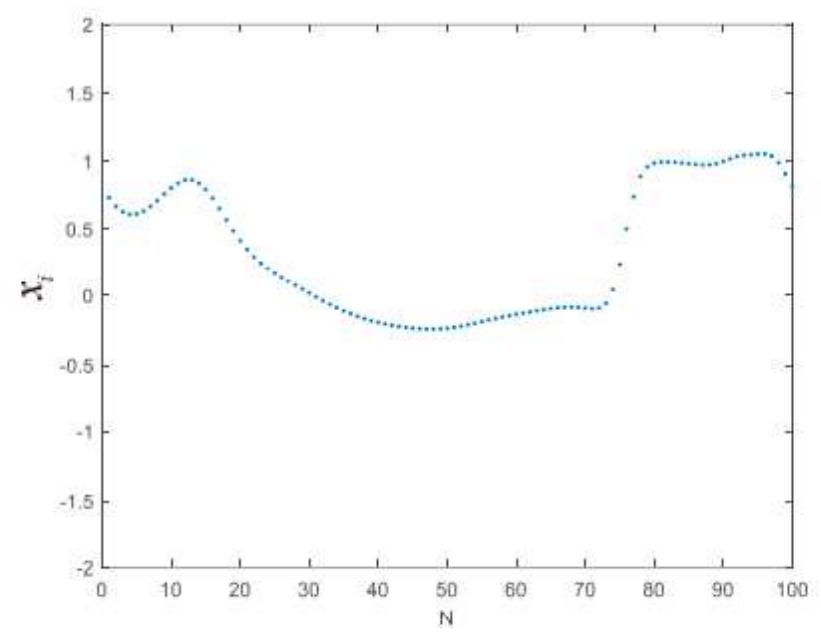

(d)

Figure 19

Please see the Manuscript PDF file for the complete figure caption 


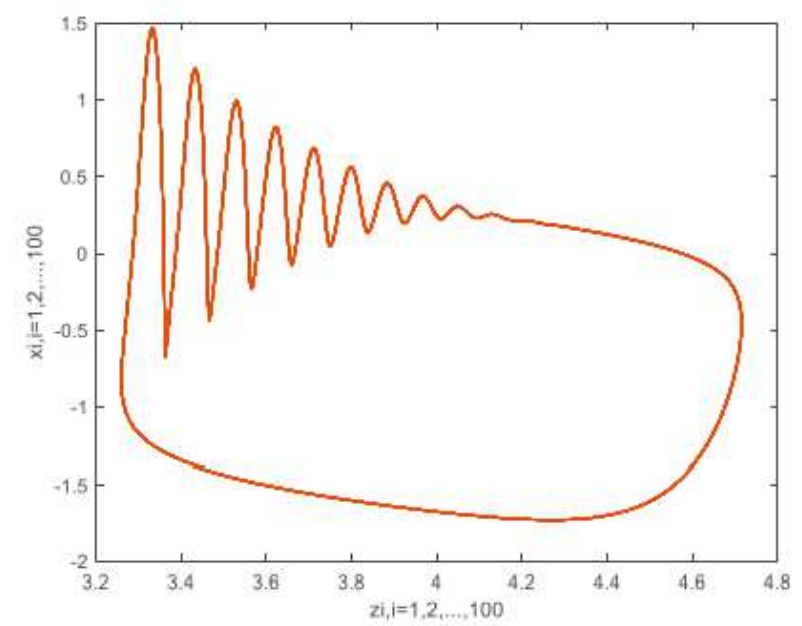

(a)

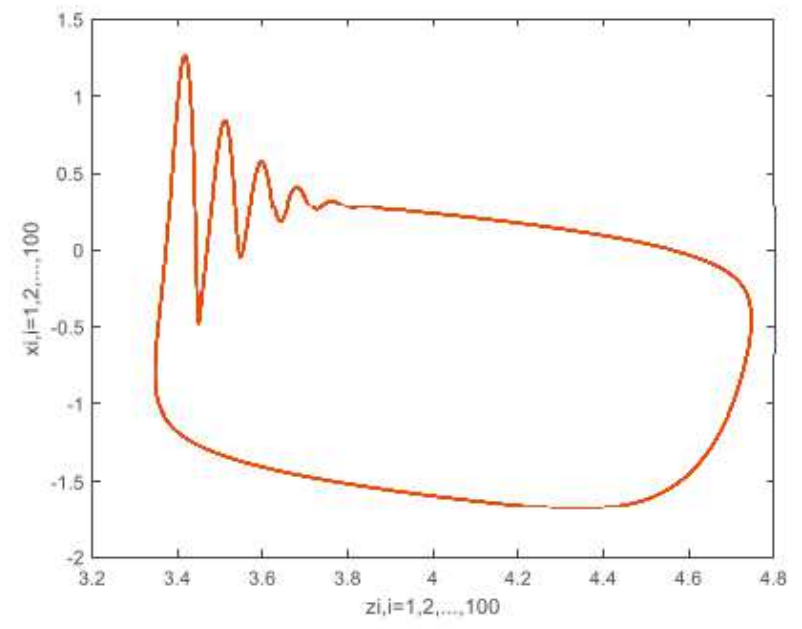

(c)

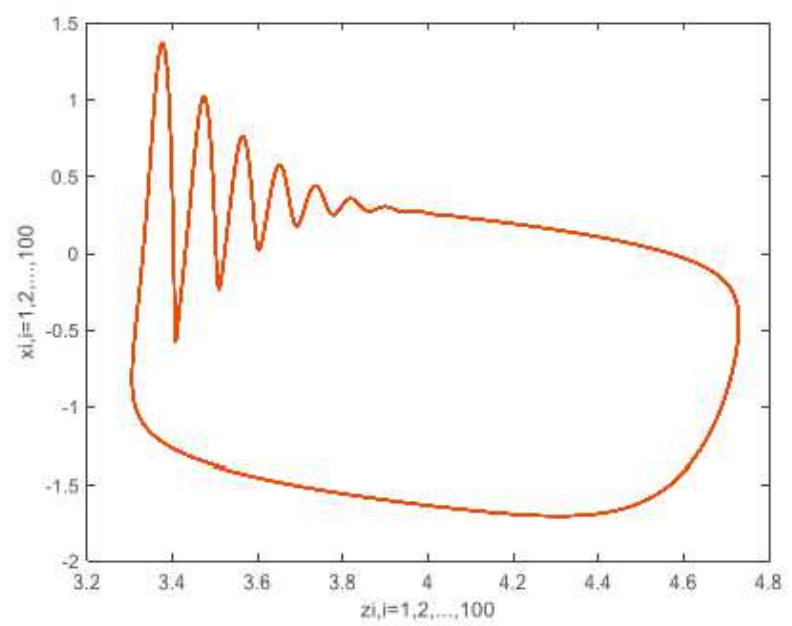

(b)

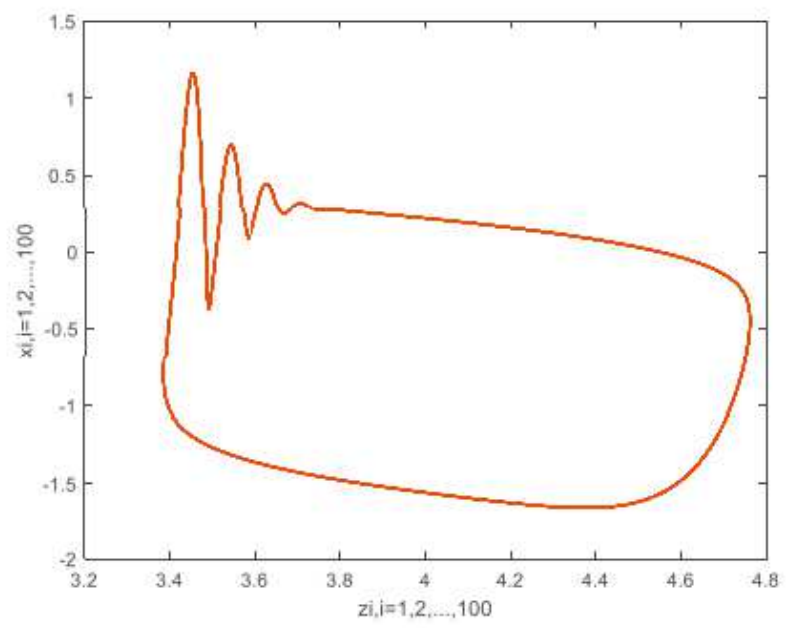

(d)

Figure 20

Please see the Manuscript PDF file for the complete figure caption 


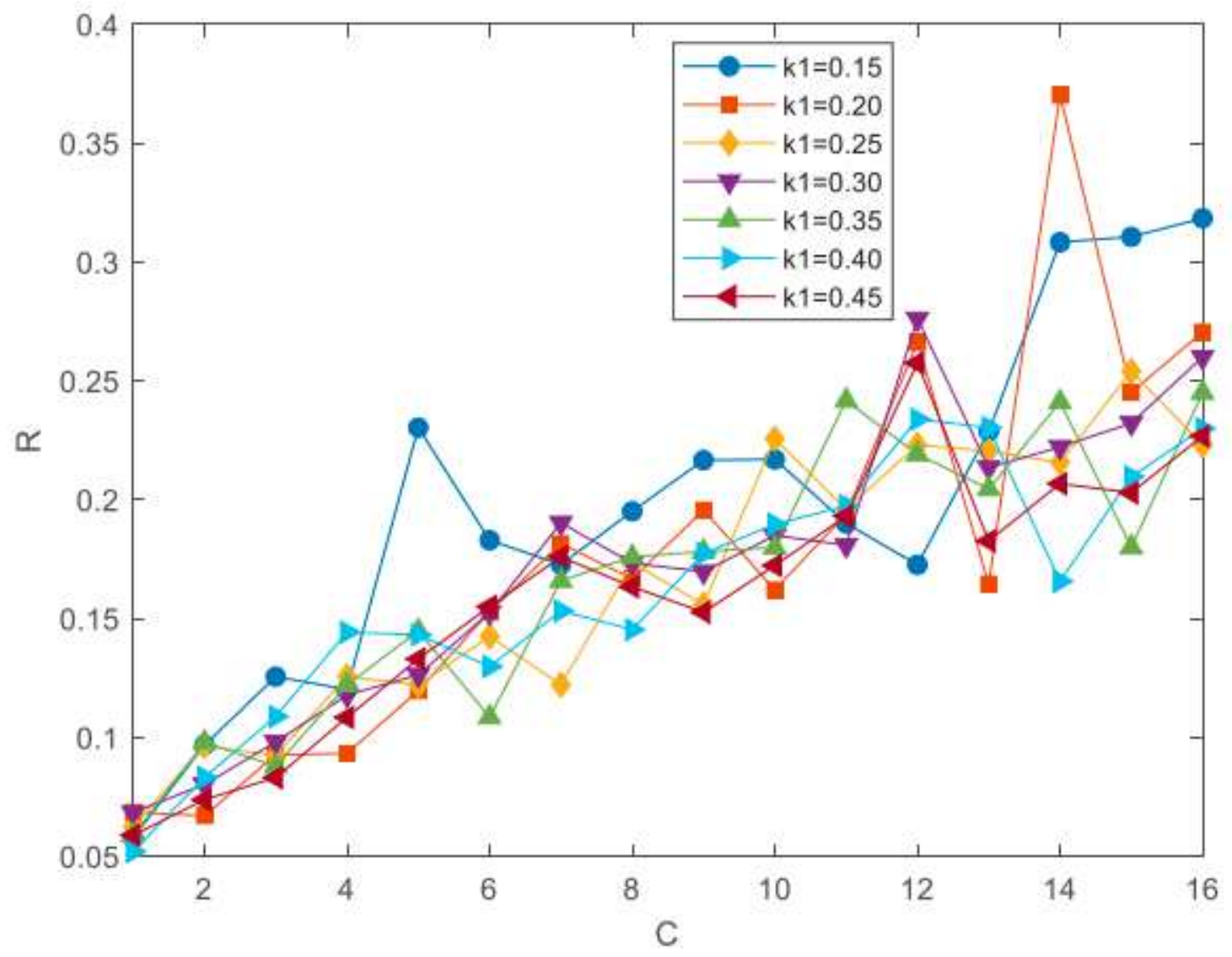

Figure 21

Please see the Manuscript PDF file for the complete figure caption 


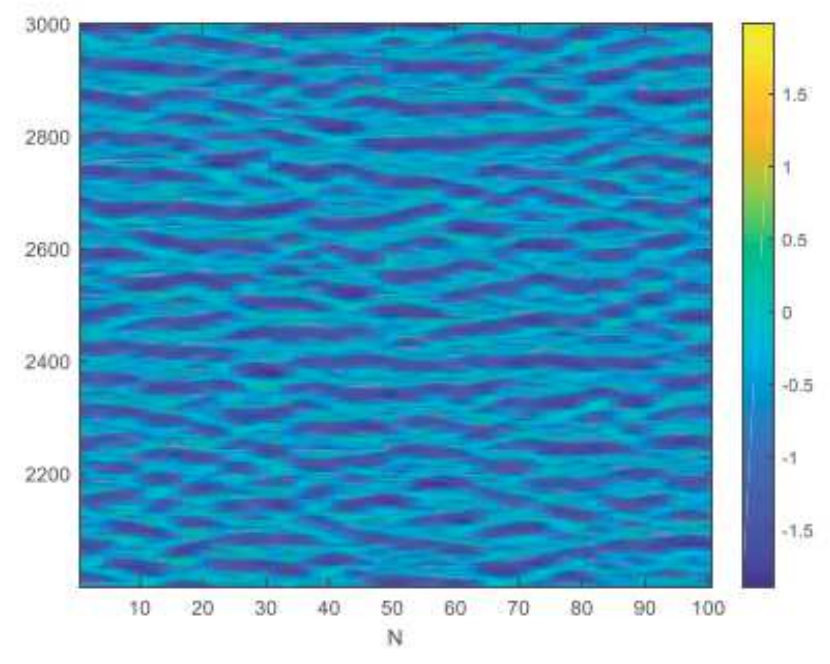

(a)

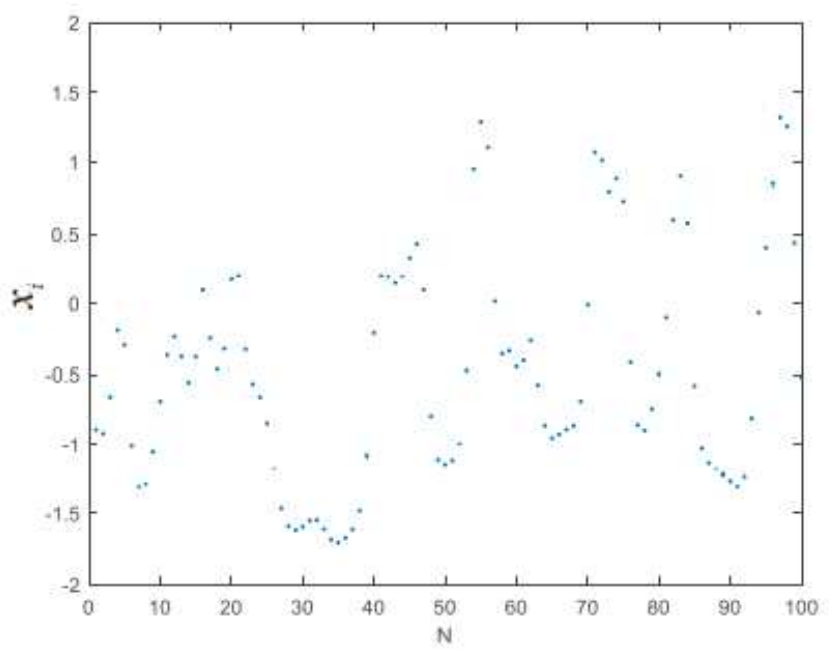

(c)

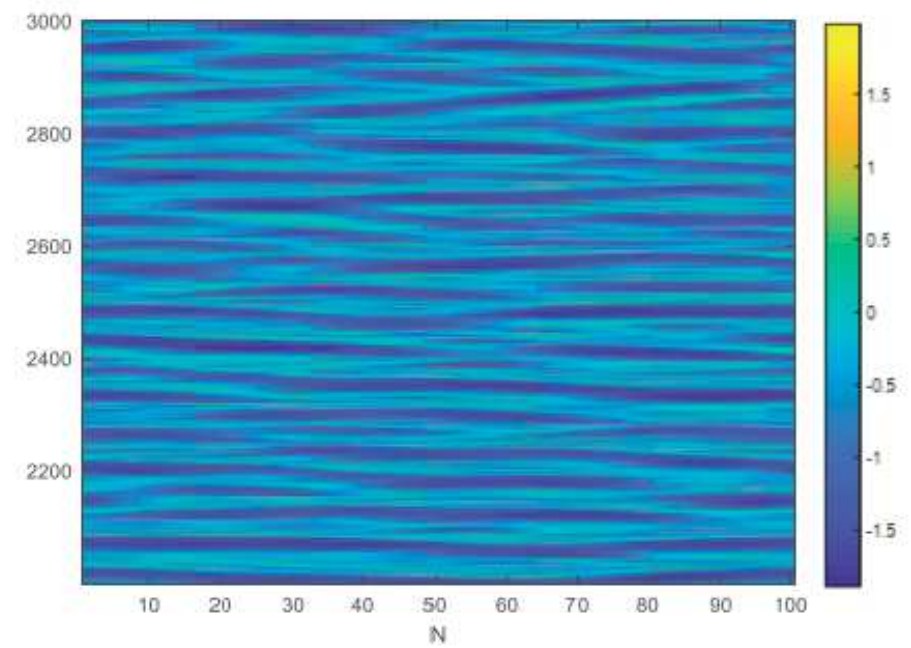

(b)

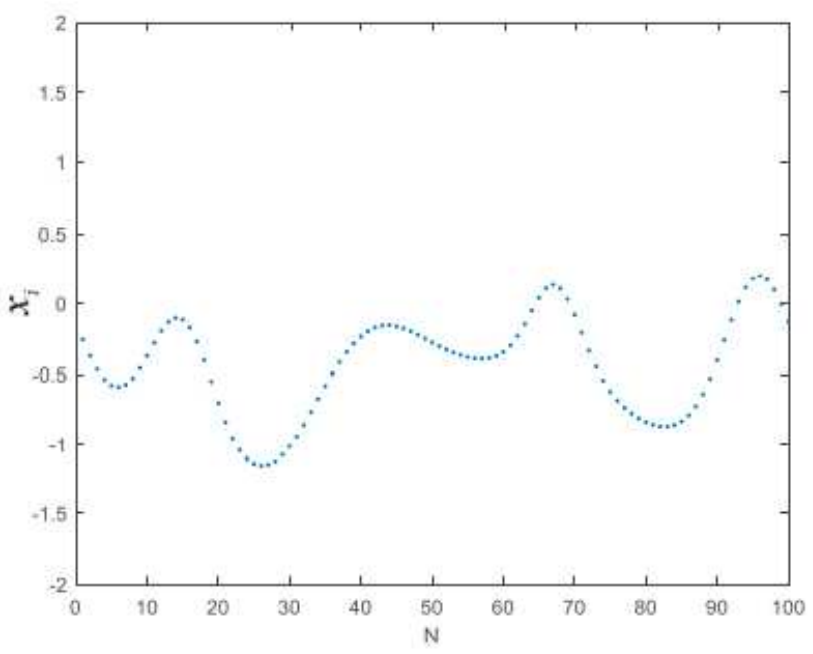

(d)

Figure 22

Please see the Manuscript PDF file for the complete figure caption 


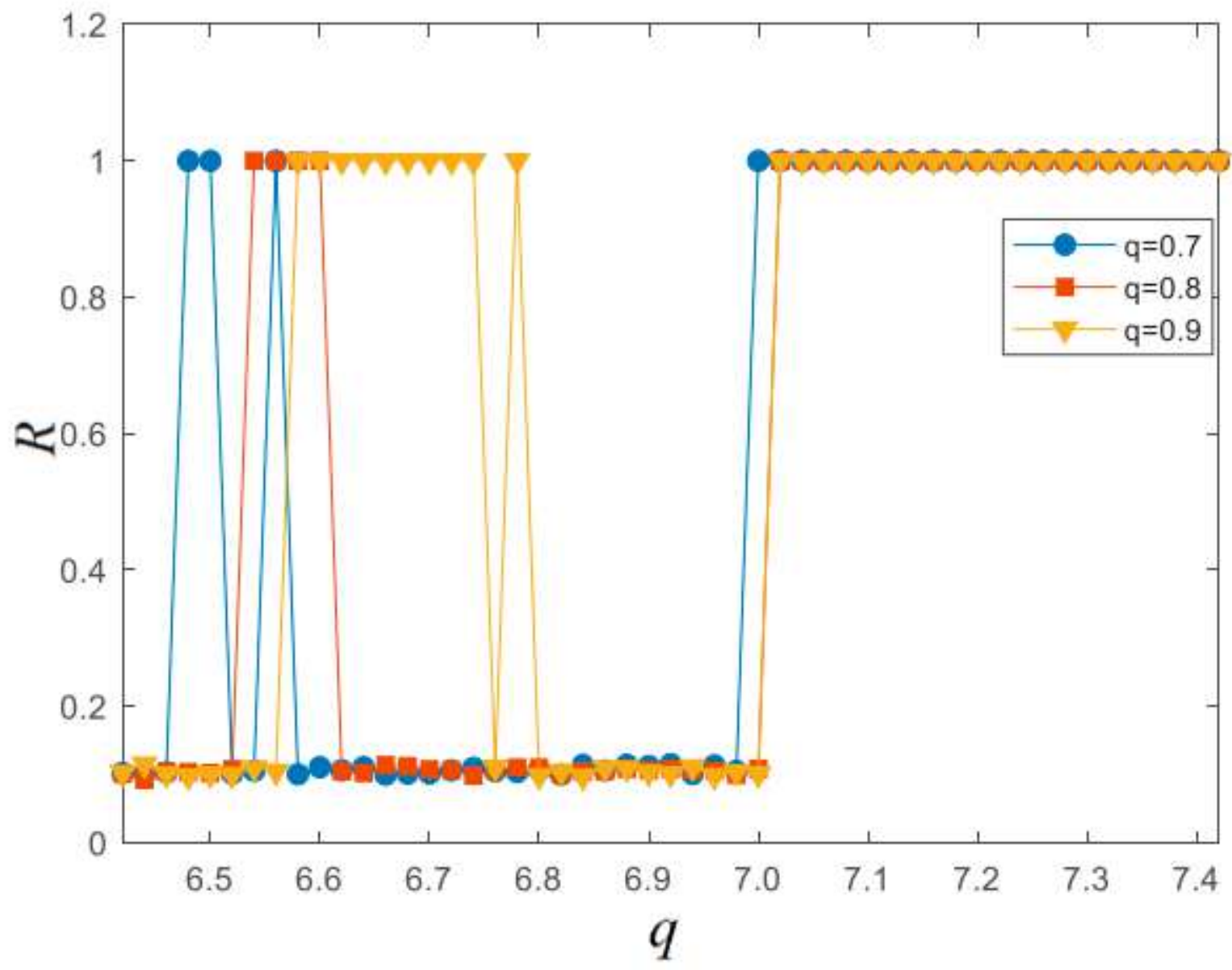

Figure 23

Please see the Manuscript PDF file for the complete figure caption 


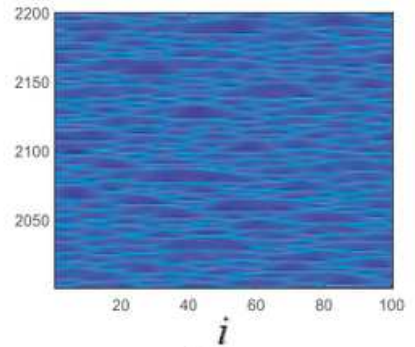

(a)

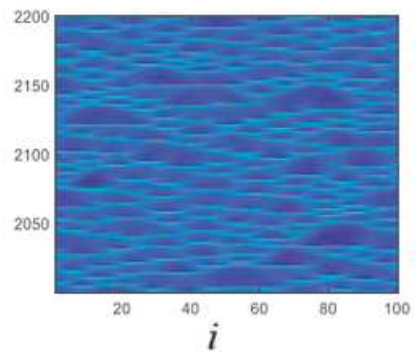

(e)

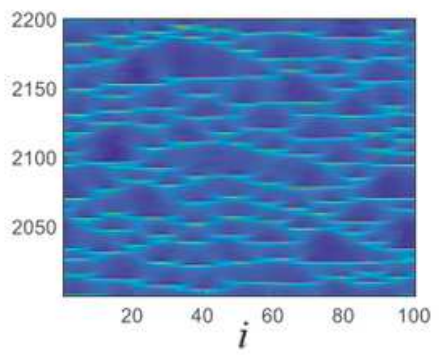

(i)

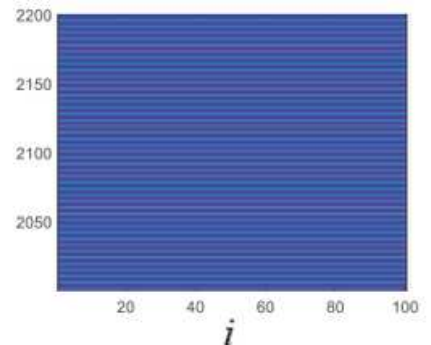

(b)

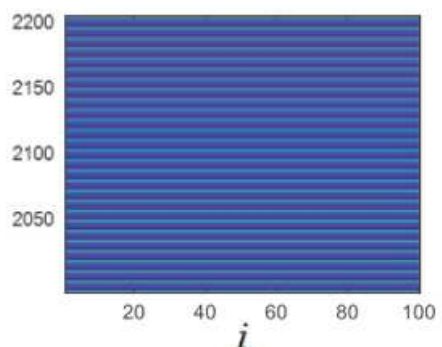

(f)

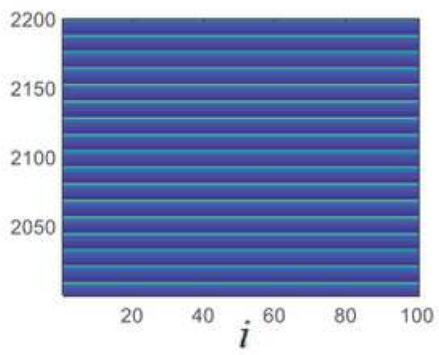

(j)

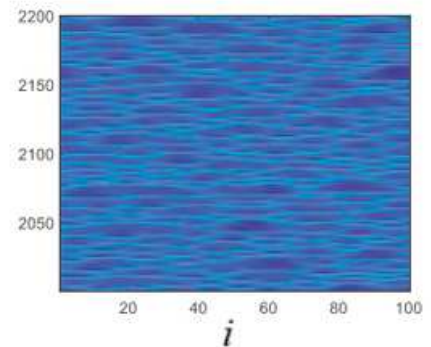

(c)

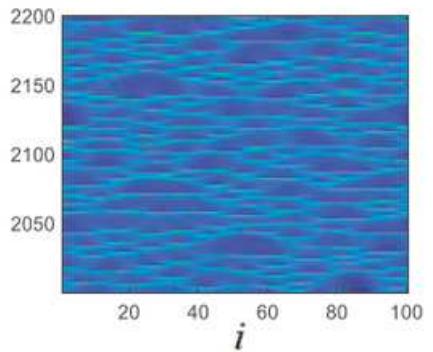

(g)

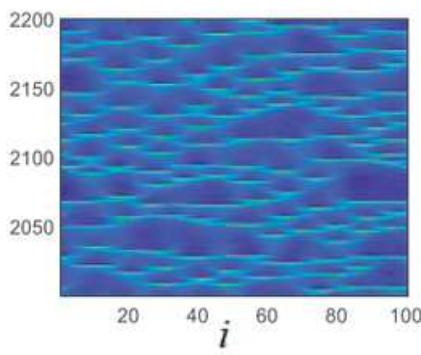

(k)

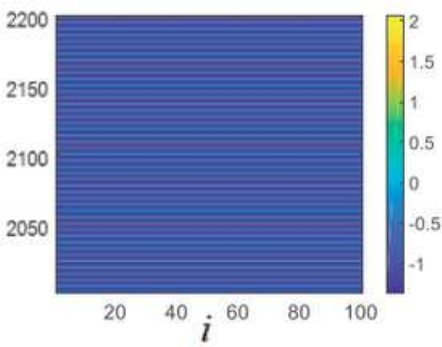

(d)

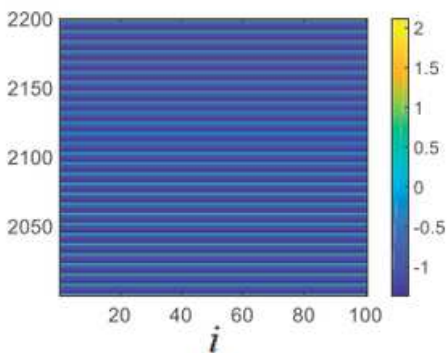

(h)

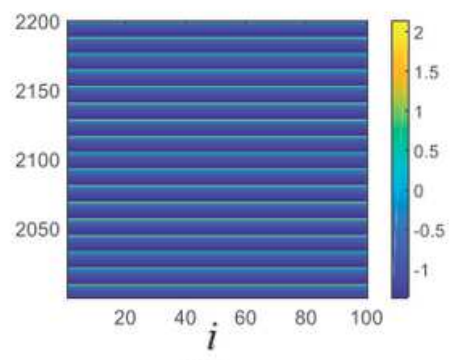

(1)

Figure 24

Please see the Manuscript PDF file for the complete figure caption 


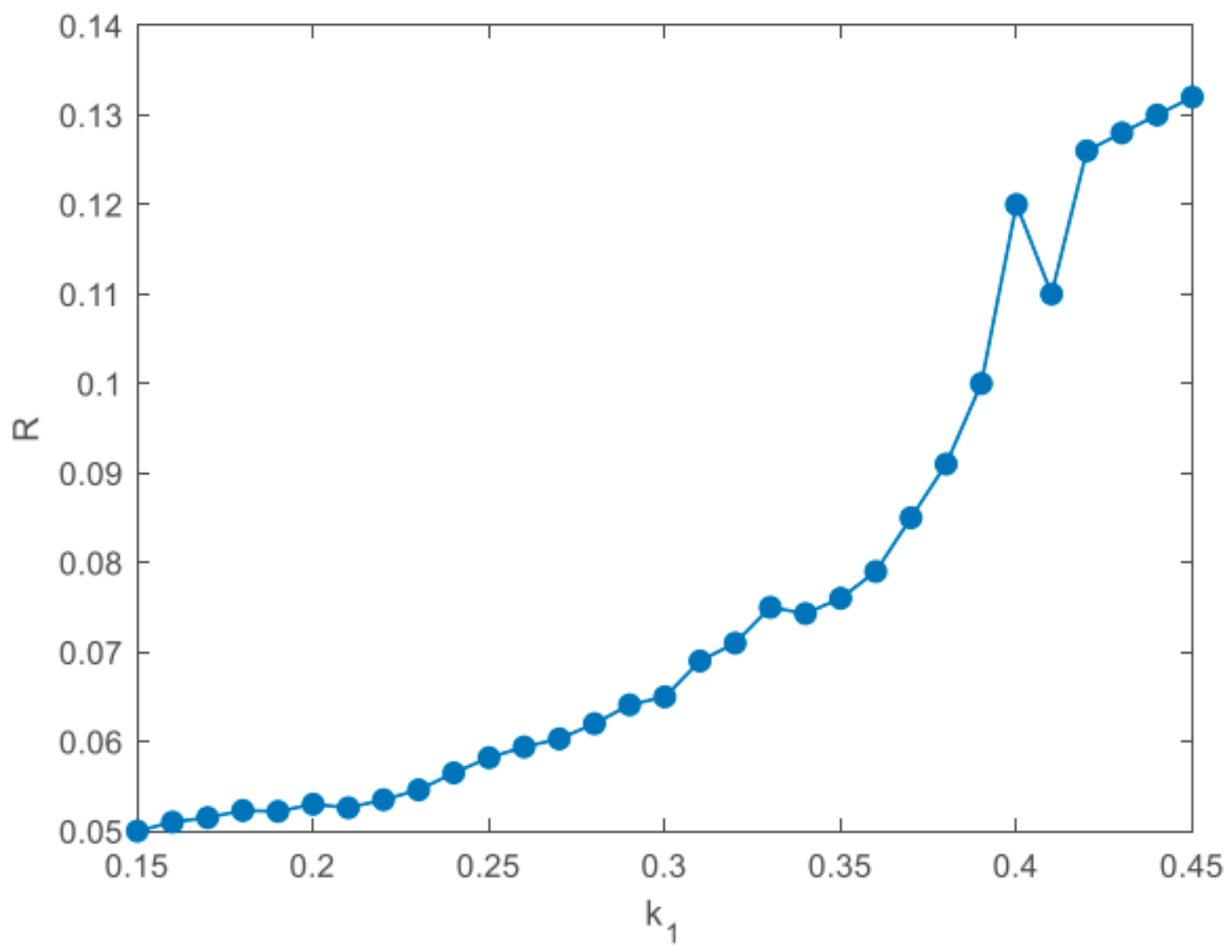

Figure 25

Please see the Manuscript PDF file for the complete figure caption 